\title{
An Efficient Computational Approach for the Calculation of the Vibrational Density of States
}

Chiara Aieta, $\uparrow$ Fabio Gabas, $\ddagger$ and Michele Ceotto $*, \emptyset$

Dipartimento di Chimica, Università degli Studi di Milano, via C. Golgi 19, 20133 Milano, Italy, Dipartimento di Chimica, Università degli Studi di Milano, via C. Golgi 19, 20133 Milano, Italy and CINECA - Interuniversity Computing Center Supercomputing Applications and Innovation Department - SCAI Via R. Sanzio, 4 - 20090 Segrate (MI) - Italy, and Dipartimento di Chimica, Università degli Studi di Milano, via C. Golgi 19, Tel: +39 0250314258, 20133 Milano, Italy

E-mail: michele.ceotto@unimi.it

\section{Supporting Information}

\section{Harmonic Frequencies and Anharmonic Constants Used}

Input data including harmonic frequencies, X-matrix, and separable degrees of freedom in the input format of program paradensum for all the molecules treated in the paper are given below.

$\mathrm{H} 2 \mathrm{O}$

H2O from Exptl. data from Benedict et al., J. Chem. Phys. 1956, 24, 1139-1165

3, 0, 0, 'We'

3813.085

1646.495

3944.140

lower

$-42.58$

$-15.93-16.81$

$\begin{array}{lll}-165.82 & -20.32 & -47.57\end{array}$ 
trans-HOCO

At CCSD(T)/ANO1 level of theory

6, 0, 0, "We"

534.3400302

619.096215

1082.73427

1255.703066

1890.429517

3829.676649

lower

$-10.4901$

$3.8013-0.1334$

$-5.1221-8.1088-8.6805$

$-2.7016-1.7113-10.0615-11.5228$

$\begin{array}{lllll}-1.8307 & -5.9370 & -14.2017 & -5.6786 & -12.9506\end{array}$

$\begin{array}{llllll}-9.6966 & -2.1636 & -4.6726 & -19.2512 & -3.4919 & -84.6154\end{array}$

7 qro 3.07483911 


\begin{tabular}{|c|c|c|c|c|c|c|c|c|c|c|}
\hline \multicolumn{11}{|c|}{$\mathrm{CH} 2=\mathrm{NCH} 3$} \\
\hline \multicolumn{11}{|c|}{ 18, 0, 0, "We" } \\
\hline \multicolumn{11}{|c|}{226.2023} \\
\hline \multicolumn{11}{|c|}{494.2954} \\
\hline \multicolumn{11}{|c|}{752.5276} \\
\hline \multicolumn{11}{|c|}{1021.8283} \\
\hline \multicolumn{11}{|c|}{1209.7171} \\
\hline \multicolumn{11}{|c|}{1237.3463} \\
\hline \multicolumn{11}{|c|}{1268.6819} \\
\hline \multicolumn{11}{|c|}{1368.5621} \\
\hline \multicolumn{11}{|c|}{1606.2200} \\
\hline \multicolumn{11}{|c|}{1639.7899} \\
\hline \multicolumn{11}{|c|}{1642.0379} \\
\hline \multicolumn{11}{|c|}{1668.0832} \\
\hline \multicolumn{11}{|c|}{1900.7554} \\
\hline \multicolumn{11}{|c|}{3157.6598} \\
\hline \multicolumn{11}{|c|}{3225.7018} \\
\hline \multicolumn{11}{|c|}{3257.0929} \\
\hline \multicolumn{11}{|c|}{3283.7637} \\
\hline \multicolumn{11}{|c|}{3375.0422} \\
\hline lower & & & & & & & & & & \\
\hline-53.243 & & & & & & & & & & \\
\hline 0.952 & -32.425 & & & & & & & & & \\
\hline 0.728 & -100.491 & -21.618 & & & & & & & & \\
\hline-37.197 & 0.533 & -0.410 & -52.907 & & & & & & & \\
\hline 0.594 & -13.372 & -51.253 & -0.903 & -42.456 & & & & & & \\
\hline-2.702 & -1.216 & -1.167 & -4.263 & 1.047 & -7.198 & & & & & \\
\hline-4.369 & -12.704 & -18.944 & -15.505 & -14.346 & & -1.173 & 0.593 & & & \\
\hline 0.073 & -13.592 & -15.215 & -0.200 & -38.312 & -0.271 & -0.579 & 1.052 & & & \\
\hline-9.259 & -8.196 & -22.839 & -26.267 & -8.205 & -2.936 & 6.292 & -0.547 & 1.114 & & \\
\hline-0.189 & -9.548 & 17.469 & -0.219 & -66.284 & -0.924 & 0.623 & -2.844 & 0.129 & -0.077 & \\
\hline-9.554 & -0.044 & 0.587 & -8.703 & -3.009 & 0.248 & -5.694 & -1.860 & -6.349 & -2.484 & -0.872 \\
\hline-0.694 & -3.298 & -6.580 & -0.741 & 0.023 & -2.436 & -2.209 & -7.132 & -0.678 & -9.949 & -0.774 \\
\hline-1.582 & & & & & & & & & & \\
\hline 0.708 & -3.443 & 0.578 & 0.116 & -2.247 & -6.522 & -7.098 & -4.320 & -5.924 & -4.657 & -2.853 \\
\hline 0.231 & -1.136 & & & & & & & & & \\
\hline-15.034 & -0.029 & 0.063 & -10.351 & 0.712 & -5.802 & 0.397 & -0.398 & 1.196 & -0.577 & 2.519 \\
\hline-1.289 & 1.058 & -2.515 & & & & & & & & \\
\hline-2.007 & 2.973 & 2.604 & 1.009 & 2.890 & -8.941 & -3.153 & -1.772 & -1.998 & -6.248 & -3.505 \\
\hline-2.956 & -4.796 & -0.487 & -3.669 & & & & & & & \\
\hline-6.110 & -0.316 & -0.375 & -4.847 & 0.610 & -1.900 & 0.292 & -0.782 & 4.852 & -0.852 & -0.149 \\
\hline-0.356 & 0.154 & -3.296 & 1.899 & -2.474 & & & & & & \\
\hline-2.946 & -0.421 & -0.087 & -0.419 & 1.350 & 2.822 & 0.760 & -0.393 & -1.062 & 0.061 & -4.532 \\
\hline 1.341 & 2.894 & 0.751 & -3.340 & 1.610 & -0.569 & & & & & \\
\hline-0.588 & 0.135 & 0.873 & -0.592 & 3.533 & -0.359 & -0.955 & -1.052 & 1.219 & 0.426 & -1.025 \\
\hline 0.440 & -0.213 & -0.122 & -0.884 & -3.23 & -6.2 & 282 & & & & \\
\hline
\end{tabular}


C14H10

B3LYP/cc-pVdz

66, 0, 0, "We"

3199.0964

3198.6391

3186.5235

3186.2479

3173.6853

3172.4230

3168.3545

3167.5511

3165.9078

3159.1236

1679.1549

1678.8922

1630.7939

1603.5199

1587.4114

1517.0694

1486.3881

1476.8076

1445.2193

1426.6604

1404.0311

1386.6260

1337.5310

1293.7913

1281.7350

1280.7179

1199.6219

1181.9636

1176.5654

1164.9173

1155.0789

1124.7579

1034.1094

1029.9256

1004.3196

1003.6768

980.7665

975.9614

932.1833

923.7084

913.0110

908.2774

880.6780

857.8697

824.5870

796.0553

777.2580

773.5348

764.9096

749.0457

659.4144

637.8371

614.3933 
594.3062

532.9688

512.2589

490.4809

484.8157

398.0895

394.7275

390.5074

273.3717

239.2558

235.5208

122.8895

93.2344

lower

$-0.117060 \mathrm{D}+02$

$-0.475390 \mathrm{D}+02-0.120575 \mathrm{D}+02$

$-0.361244 \mathrm{D}+02-0.363528 \mathrm{D}+02 \quad-0.829824 \mathrm{D}+01$

$-0.371074 \mathrm{D}+02-0.373670 \mathrm{D}+02 \quad-0.335788 \mathrm{D}+02 \quad-0.848058 \mathrm{D}+01$

$-0.174615 \mathrm{D}+02-0.169810 \mathrm{D}+02 \quad-0.273028 \mathrm{D}+02 \quad-0.267019 \mathrm{D}+02-0.980424 \mathrm{D}+01$

$-0.183825 \mathrm{D}+02 \quad-0.178129 \mathrm{D}+02 \quad-0.301799 \mathrm{D}+02 \quad-0.295119 \mathrm{D}+02 \quad-0.426891 \mathrm{D}+02 \quad-0.122058 \mathrm{D}+02$

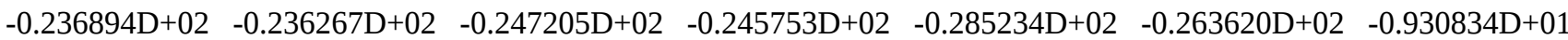

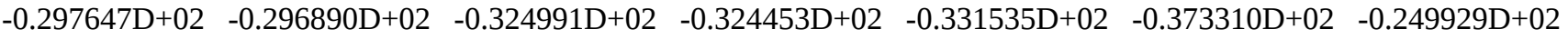
$-0.852131 \mathrm{D}+01$

$-0.806004 \mathrm{D}+01 \quad-0.776044 \mathrm{D}+01 \quad-0.105506 \mathrm{D}+02 \quad-0.984768 \mathrm{D}+01 \quad-0.269303 \mathrm{D}+02 \quad-0.133855 \mathrm{D}+02 \quad-0.486148 \mathrm{D}+02$ $-0.112856 \mathrm{D}+02-0.303433 \mathrm{D}+02$

$-0.798367 \mathrm{D}+00 \quad-0.581462 \mathrm{D}+00 \quad-0.147994 \mathrm{D}+01 \quad-0.102007 \mathrm{D}+01 \quad-0.712021 \mathrm{D}+01 \quad-0.175798 \mathrm{D}+01-0.458874 \mathrm{D}+01$ $-0.128669 \mathrm{D}+01-0.101244 \mathrm{D}+02-0.600472 \mathrm{D}+02$

$0.905320 \mathrm{D}+00 \quad-0.222654 \mathrm{D}+01 \quad 0.736799 \mathrm{D}+00 \quad 0.287882 \mathrm{D}+01 \quad 0.103319 \mathrm{D}+01 \quad 0.451640 \mathrm{D}+01 \quad 0.149445 \mathrm{D}+01$ $-0.822199 \mathrm{D}+00 \quad-0.319825 \mathrm{D}+01 \quad-0.301005 \mathrm{D}+00 \quad-0.950911 \mathrm{D}+00$

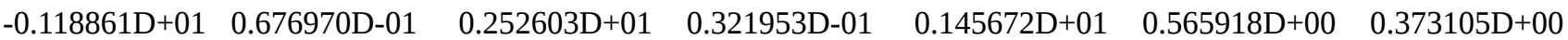
$0.721485 \mathrm{D}+00 \quad 0.115486 \mathrm{D}+01 \quad 0.251210 \mathrm{D}+01 \quad-0.299248 \mathrm{D}+01 \quad-0.122997 \mathrm{D}+01$

$-0.313875 \mathrm{D}+01 \quad-0.192587 \mathrm{D}+00 \quad-0.483596 \mathrm{D}+01 \quad-0.233094 \mathrm{D}+01 \quad 0.170311 \mathrm{D}+01 \quad 0.185904 \mathrm{D}+01 \quad 0.548155 \mathrm{D}+01$ $-0.311583 \mathrm{D}+00 \quad-0.172964 \mathrm{D}+01 \quad-0.162906 \mathrm{D}-01 \quad-0.200616 \mathrm{D}+01 \quad-0.210957 \mathrm{D}+01 \quad-0.100166 \mathrm{D}+00$

$0.167750 \mathrm{D}+01 \quad 0.973454 \mathrm{D}+00 \quad-0.161268 \mathrm{D}+01 \quad-0.477657 \mathrm{D}+01 \quad 0.267914 \mathrm{D}+01 \quad 0.363059 \mathrm{D}+00 \quad 0.159938 \mathrm{D}+01$ $0.122294 \mathrm{D}+01 \quad-0.449104 \mathrm{D}-01 \quad-0.101112 \mathrm{D}+01 \quad-0.520059 \mathrm{D}+00 \quad-0.101105 \mathrm{D}+01 \quad 0.128900 \mathrm{D}+01 \quad-$ $0.127481 \mathrm{D}+01$

$0.111586 \mathrm{D}+01 \quad 0.248767 \mathrm{D}+01 \quad-0.589315 \mathrm{D}+01 \quad-0.414951 \mathrm{D}+00 \quad 0.841735 \mathrm{D}+00 \quad-0.605012 \mathrm{D}+00 \quad-0.313644 \mathrm{D}+01$ $0.125708 \mathrm{D}+01 \quad-0.106291 \mathrm{D}+01 \quad-0.673174 \mathrm{D}+01 \quad-0.710836 \mathrm{D}-01 \quad-0.267487 \mathrm{D}+00 \quad 0.659469 \mathrm{D}+01$ $-0.185503 \mathrm{D}+01 \quad 0.870295 \mathrm{D}+00$

0.197719D+01 $\quad 0.202511 \mathrm{D}+01 \quad 0.577537 \mathrm{D}+00 \quad 0.361624 \mathrm{D}+00 \quad 0.355512 \mathrm{D}+01 \quad 0.226357 \mathrm{D}+01 \quad-0.798041 \mathrm{D}+00$

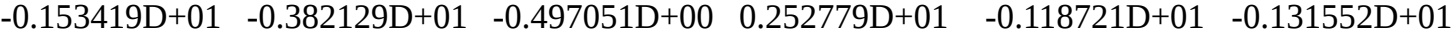

$-0.632401 \mathrm{D}+01-0.553638 \mathrm{D}+01-0.155566 \mathrm{D}+01$ 
$0.257815 \mathrm{D}+01 \quad 0.203574 \mathrm{D}+01 \quad 0.131473 \mathrm{D}+01 \quad 0.133253 \mathrm{D}+01 \quad-0.648106 \mathrm{D}+00 \quad 0.712673 \mathrm{D}+00 \quad 0.238287 \mathrm{D}+01$ $0.125068 \mathrm{D}+01 \quad 0.381575 \mathrm{D}+00 \quad-0.499527 \mathrm{D}-01 \quad-0.596039 \mathrm{D}+01 \quad-0.353935 \mathrm{D}+01 \quad-0.562787 \mathrm{D}+01$ $-0.470421 \mathrm{D}+01 \quad-0.561394 \mathrm{D}+01 \quad-0.382387 \mathrm{D}+01 \quad-0.102003 \mathrm{D}+01$

$-0.224777 \mathrm{D}+00 \quad-0.238412 \mathrm{D}+00 \quad 0.190062 \mathrm{D}+01 \quad 0.253441 \mathrm{D}+01 \quad 0.264207 \mathrm{D}+01 \quad 0.391612 \mathrm{D}+01 \quad 0.399210 \mathrm{D}+01$ $-0.160876 \mathrm{D}+01 \quad 0.324500 \mathrm{D}+00 \quad 0.295164 \mathrm{D}+00 \quad-0.545585 \mathrm{D}+01 \quad-0.401274 \mathrm{D}+01 \quad-0.760685 \mathrm{D}+01$ $-0.376959 \mathrm{D}+01 \quad-0.293684 \mathrm{D}+01-0.516254 \mathrm{D}+01 \quad-0.204453 \mathrm{D}+01 \quad-0.167721 \mathrm{D}+01$

$0.866610 \mathrm{D}+00 \quad 0.727145 \mathrm{D}+00 \quad 0.310018 \mathrm{D}+01 \quad 0.256239 \mathrm{D}+01 \quad 0.119238 \mathrm{D}+01 \quad 0.130860 \mathrm{D}+01 \quad 0.842573 \mathrm{D}+00$ $0.147816 \mathrm{D}+01 \quad 0.373147 \mathrm{D}-01 \quad-0.723184 \mathrm{D}+00 \quad-0.777302 \mathrm{D}+01 \quad-0.502999 \mathrm{D}+01 \quad-0.383693 \mathrm{D}+01$

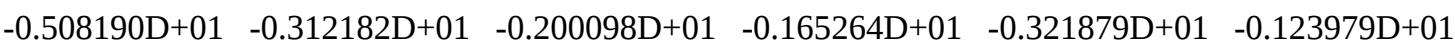

$0.208071 \mathrm{D}+00 \quad 0.267207 \mathrm{D}+00 \quad-0.763843 \mathrm{D}-01 \quad-0.160404 \mathrm{D}-01 \quad-0.917007 \mathrm{D}+00 \quad-0.737360 \mathrm{D}+00 \quad-0.760397 \mathrm{D}+00$ $-0.268565 \mathrm{D}+00 \quad-0.164577 \mathrm{D}+01 \quad-0.183729 \mathrm{D}+01 \quad-0.165192 \mathrm{D}+01 \quad-0.216007 \mathrm{D}+01 \quad-0.261308 \mathrm{D}+01$

$-0.445509 \mathrm{D}+01 \quad-0.183321 \mathrm{D}+01 \quad-0.887796 \mathrm{D}+00 \quad-0.119542 \mathrm{D}+01 \quad-0.632082 \mathrm{D}+00 \quad-0.445924 \mathrm{D}+01 \quad-0.137535 \mathrm{D}+01$

$-0.152985 \mathrm{D}+01 \quad-0.149412 \mathrm{D}+01 \quad-0.586696 \mathrm{D}+00 \quad-0.582739 \mathrm{D}+00 \quad 0.219236 \mathrm{D}+01 \quad 0.329788 \mathrm{D}+01 \quad 0.495798 \mathrm{D}+00$ $0.465643 \mathrm{D}+00 \quad 0.883159 \mathrm{D}+00 \quad 0.833131 \mathrm{D}+00 \quad-0.714819 \mathrm{D}+01 \quad-0.488120 \mathrm{D}+01 \quad-0.378933 \mathrm{D}+01$

$-0.316343 \mathrm{D}+01 \quad-0.395478 \mathrm{D}+01 \quad-0.381942 \mathrm{D}+01 \quad-0.311070 \mathrm{D}+01 \quad-0.323053 \mathrm{D}+01 \quad-0.159863 \mathrm{D}+01 \quad-0.687318 \mathrm{D}+00$ $-0.825986 \mathrm{D}+00$

$0.571496 \mathrm{D}+00 \quad 0.675235 \mathrm{D}+00 \quad 0.140303 \mathrm{D}+01 \quad 0.151359 \mathrm{D}+01 \quad 0.709448 \mathrm{D}+00 \quad 0.444104 \mathrm{D}+00 \quad 0.209760 \mathrm{D}+01$ $0.357552 \mathrm{D}+00 \quad 0.341876 \mathrm{D}+01 \quad 0.621639 \mathrm{D}+01 \quad-0.426919 \mathrm{D}+01 \quad-0.741065 \mathrm{D}+01 \quad-0.849416 \mathrm{D}+01$

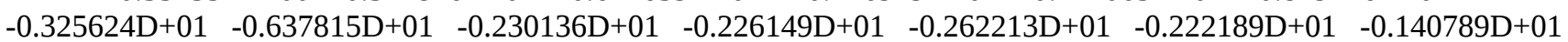
$-0.195824 \mathrm{D}+01-0.104252 \mathrm{D}+01$

$-0.107963 \mathrm{D}+01 \quad-0.108543 \mathrm{D}+01 \quad-0.648459 \mathrm{D}+00 \quad-0.672728 \mathrm{D}+00 \quad 0.482728 \mathrm{D}+00 \quad 0.573603 \mathrm{D}+00 \quad-0.277694 \mathrm{D}+00$ $\begin{array}{llllll}-0.200025 \mathrm{D}+00 & -0.409624 \mathrm{D}+00 & -0.642362 \mathrm{D}+00 & -0.253421 \mathrm{D}+01 & -0.321486 \mathrm{D}+01 & -0.315610 \mathrm{D}+01\end{array}$

$-0.202928 \mathrm{D}+01-0.147235 \mathrm{D}+01 \quad-0.156105 \mathrm{D}+01-0.140108 \mathrm{D}+01 \quad-0.142971 \mathrm{D}+01 \quad-0.227397 \mathrm{D}+01 \quad-0.148701 \mathrm{D}+01$ $-0.112578 \mathrm{D}+01-0.176821 \mathrm{D}+01-0.725579 \mathrm{D}+00$

$-0.649643 \mathrm{D}+00 \quad-0.639486 \mathrm{D}+00 \quad-0.854279 \mathrm{D}+00 \quad-0.850603 \mathrm{D}+00 \quad-0.792462 \mathrm{D}+00 \quad-0.911237 \mathrm{D}+00 \quad-0.281398 \mathrm{D}+00$

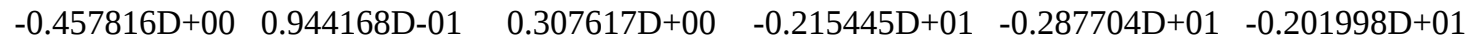

$-0.105251 \mathrm{D}+01-0.158984 \mathrm{D}+01 \quad-0.200554 \mathrm{D}+01 \quad-0.194083 \mathrm{D}+01 \quad-0.145166 \mathrm{D}+01 \quad-0.789920 \mathrm{D}+00 \quad-0.109627 \mathrm{D}+01$ $-0.938399 \mathrm{D}+00-0.171383 \mathrm{D}+01 \quad-0.113511 \mathrm{D}+01-0.615205 \mathrm{D}+00$

$-0.296887 \mathrm{D}+00 \quad-0.264739 \mathrm{D}+00 \quad-0.637000 \mathrm{D}+00 \quad-0.566080 \mathrm{D}+00 \quad-0.160441 \mathrm{D}+01 \quad-0.100230 \mathrm{D}+01 \quad-0.208330 \mathrm{D}+01$ $-0.757317 \mathrm{D}+00 \quad-0.383034 \mathrm{D}+01-0.541975 \mathrm{D}+01 \quad-0.707176 \mathrm{D}+00 \quad-0.273666 \mathrm{D}+01-0.254761 \mathrm{D}+01$

$-0.548349 \mathrm{D}+00 \quad-0.132041 \mathrm{D}+01 \quad-0.638816 \mathrm{D}+00 \quad-0.943339 \mathrm{D}+00 \quad-0.712947 \mathrm{D}+00 \quad-0.918377 \mathrm{D}+00 \quad-0.131401 \mathrm{D}+01$

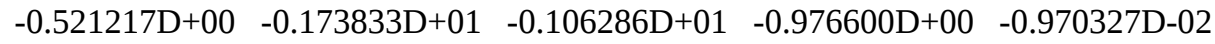

$-0.952276 \mathrm{D}+00 \quad-0.921669 \mathrm{D}+00 \quad-0.144416 \mathrm{D}+01 \quad-0.140972 \mathrm{D}+01 \quad-0.169899 \mathrm{D}+01-0.185709 \mathrm{D}+01-0.141021 \mathrm{D}+01$ $-0.171267 \mathrm{D}+01 \quad-0.967657 \mathrm{D}+00 \quad-0.701703 \mathrm{D}+00 \quad-0.209182 \mathrm{D}+01-0.203625 \mathrm{D}+01 \quad-0.932319 \mathrm{D}+00$

$-0.863193 \mathrm{D}+00 \quad-0.659068 \mathrm{D}+00 \quad-0.115595 \mathrm{D}+01 \quad-0.106052 \mathrm{D}+01 \quad-0.161275 \mathrm{D}+01 \quad-0.153521 \mathrm{D}+01 \quad-0.134797 \mathrm{D}+01$

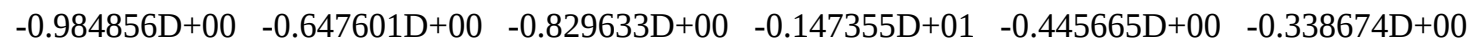

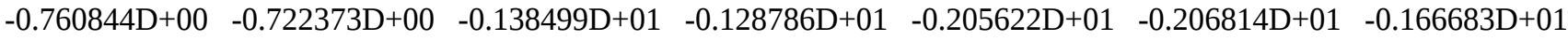
$-0.163184 \mathrm{D}+01-0.141899 \mathrm{D}+01-0.150029 \mathrm{D}+01 \quad-0.185684 \mathrm{D}+01-0.187902 \mathrm{D}+01 \quad-0.146175 \mathrm{D}+01$

$-0.417183 \mathrm{D}+00 \quad-0.107642 \mathrm{D}+01 \quad-0.122567 \mathrm{D}+01-0.879790 \mathrm{D}+00 \quad-0.155187 \mathrm{D}+01 \quad-0.946453 \mathrm{D}+00 \quad-0.761328 \mathrm{D}+00$

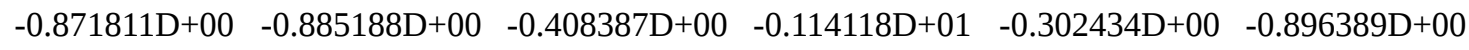

$-0.461440 \mathrm{D}-01$

$-0.148053 \mathrm{D}+01 \quad-0.147670 \mathrm{D}+01 \quad-0.106707 \mathrm{D}+01 \quad-0.107669 \mathrm{D}+01 \quad-0.755776 \mathrm{D}+00 \quad-0.404613 \mathrm{D}+00 \quad-0.142114 \mathrm{D}+01$ $-0.876215 \mathrm{D}+00 \quad-0.186735 \mathrm{D}+01 \quad-0.215677 \mathrm{D}+01 \quad-0.155047 \mathrm{D}+01-0.224474 \mathrm{D}+01 \quad-0.175989 \mathrm{D}+01$

$-0.122359 \mathrm{D}+01 \quad 0.511511 \mathrm{D}+00 \quad-0.148714 \mathrm{D}+01 \quad-0.169265 \mathrm{D}+01 \quad-0.977918 \mathrm{D}+00 \quad-0.991571 \mathrm{D}+00 \quad-0.772163 \mathrm{D}+00$

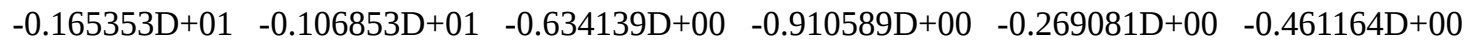


$-0.646691 \mathrm{D}-01 \quad-0.991723 \mathrm{D}-01$

$-0.257158 \mathrm{D}+01 \quad-0.264116 \mathrm{D}+01 \quad-0.195068 \mathrm{D}+01 \quad-0.201972 \mathrm{D}+01 \quad-0.143349 \mathrm{D}+01 \quad-0.153605 \mathrm{D}+01-0.172418 \mathrm{D}+01$ $-0.218465 \mathrm{D}+01-0.636868 \mathrm{D}+00 \quad-0.110835 \mathrm{D}+00 \quad-0.202668 \mathrm{D}+01-0.122262 \mathrm{D}+01 \quad-0.148445 \mathrm{D}+01$

$-0.143982 \mathrm{D}+01 \quad-0.184822 \mathrm{D}+01 \quad-0.191409 \mathrm{D}+01 \quad-0.167494 \mathrm{D}+01 \quad-0.164579 \mathrm{D}+01 \quad-0.913748 \mathrm{D}+00 \quad-0.202790 \mathrm{D}+00$ $\begin{array}{llllll}-0.211128 \mathrm{D}+01 & -0.583976 \mathrm{D}+00 & -0.724549 \mathrm{D}+00 & -0.264188 \mathrm{D}+00 & 0.239890 \mathrm{D}+00 & -0.217464 \mathrm{D}-02\end{array}$ $0.776696 \mathrm{D}+00 \quad 0.734181 \mathrm{D}+00 \quad 0.617890 \mathrm{D}+00$

$-0.212372 \mathrm{D}+01 \quad-0.215032 \mathrm{D}+01 \quad-0.183927 \mathrm{D}+01 \quad-0.189493 \mathrm{D}+01 \quad-0.149349 \mathrm{D}+01 \quad-0.161298 \mathrm{D}+01 \quad-0.138046 \mathrm{D}+01$ $\begin{array}{llllll}-0.173476 \mathrm{D}+01 & -0.639310 \mathrm{D}+00 & -0.231572 \mathrm{D}+00 & -0.223253 \mathrm{D}+01 & -0.156725 \mathrm{D}+01 & -0.161573 \mathrm{D}+01\end{array}$

$-0.146762 \mathrm{D}+01-0.142014 \mathrm{D}+01 \quad-0.137304 \mathrm{D}+01-0.113676 \mathrm{D}+01-0.139591 \mathrm{D}+01 \quad-0.120951 \mathrm{D}+01-0.401257 \mathrm{D}+00$ $\begin{array}{llllll}-0.130528 \mathrm{D}+01 & -0.754668 \mathrm{D}+00 & -0.721245 \mathrm{D}+00 & -0.327985 \mathrm{D}+00 & 0.181102 \mathrm{D}+00 & -0.147031 \mathrm{D}+00\end{array}$ $0.493838 \mathrm{D}+00 \quad-0.965798 \mathrm{D}-01 \quad 0.648980 \mathrm{D}+00 \quad 0.748844 \mathrm{D}-01$

$-0.139867 \mathrm{D}+01-0.136938 \mathrm{D}+01-0.136216 \mathrm{D}+01-0.134211 \mathrm{D}+01 \quad-0.157689 \mathrm{D}+01-0.153046 \mathrm{D}+01-0.154718 \mathrm{D}+01$ $-0.158714 \mathrm{D}+01 \quad-0.134608 \mathrm{D}+01 \quad-0.123887 \mathrm{D}+01 \quad-0.212315 \mathrm{D}+01-0.145067 \mathrm{D}+01 \quad-0.177667 \mathrm{D}+01$

$-0.122031 \mathrm{D}+01 \quad-0.962002 \mathrm{D}+00 \quad-0.122096 \mathrm{D}+01 \quad-0.127229 \mathrm{D}+01 \quad-0.124002 \mathrm{D}+01 \quad-0.904026 \mathrm{D}+00 \quad-0.394391 \mathrm{D}+00$ $\begin{array}{llllll}-0.737825 \mathrm{D}+00 & 0.377623 \mathrm{D}+00 & -0.681876 \mathrm{D}+00 & -0.767301 \mathrm{D}+00 & -0.438757 \mathrm{D}+00 & -0.409488 \mathrm{D}+00\end{array}$ $0.214929 \mathrm{D}+00 \quad 0.432654 \mathrm{D}+00 \quad 0.157836 \mathrm{D}+01 \quad 0.553701 \mathrm{D}+00 \quad 0.281713 \mathrm{D}+00$

$-0.176217 \mathrm{D}+01 \quad-0.178383 \mathrm{D}+01 \quad-0.129115 \mathrm{D}+01 \quad-0.131306 \mathrm{D}+01 \quad-0.753895 \mathrm{D}+00 \quad-0.803802 \mathrm{D}+00 \quad-0.845818 \mathrm{D}+00$ $\begin{array}{llllll}-0.110275 \mathrm{D}+01 & -0.175445 \mathrm{D}+00 & 0.144648 \mathrm{D}+00 & -0.263004 \mathrm{D}+01 & -0.151271 \mathrm{D}+01 & -0.156935 \mathrm{D}+01\end{array}$

$-0.168766 \mathrm{D}+01 \quad-0.194130 \mathrm{D}+01 \quad-0.116198 \mathrm{D}+01 \quad-0.158722 \mathrm{D}+01 \quad-0.797255 \mathrm{D}+00 \quad-0.143229 \mathrm{D}+01 \quad-0.764220 \mathrm{D}+00$ $-0.144200 \mathrm{D}+01 \quad-0.327668 \mathrm{D}+00 \quad-0.665061 \mathrm{D}+00 \quad-0.723958 \mathrm{D}+00 \quad-0.281613 \mathrm{D}+00 \quad-0.818355 \mathrm{D}+00$

$\begin{array}{llllll}-0.457574 \mathrm{D}-01 & -0.648541 \mathrm{D}+00 & -0.101765 \mathrm{D}+00 & -0.391543 \mathrm{D}+00 & 0.261494 \mathrm{D}+00 & -0.351446 \mathrm{D}+00\end{array}$

$-0.607419 \mathrm{D}+00 \quad-0.602846 \mathrm{D}+00 \quad-0.738915 \mathrm{D}+00 \quad-0.730390 \mathrm{D}+00 \quad-0.830887 \mathrm{D}+00 \quad-0.932971 \mathrm{D}+00 \quad-0.596289 \mathrm{D}+00$ $\begin{array}{llllll}-0.791174 \mathrm{D}+00 & -0.306678 \mathrm{D}+00 & -0.974743 \mathrm{D}-01 & -0.113316 \mathrm{D}+01 & -0.556126 \mathrm{D}+00 & -0.195441 \mathrm{D}+00\end{array}$

$-0.190816 \mathrm{D}+01 \quad-0.314099 \mathrm{D}+01 \quad-0.239945 \mathrm{D}+01 \quad-0.648405 \mathrm{D}+00 \quad-0.115237 \mathrm{D}+01 \quad-0.567343 \mathrm{D}+00 \quad-0.679216 \mathrm{D}+00$

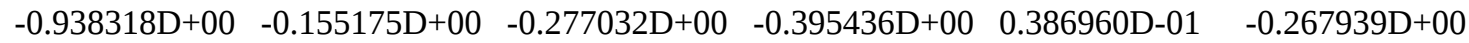

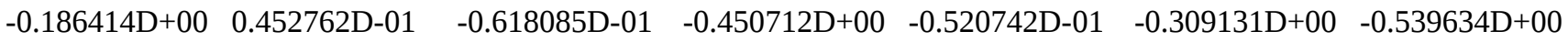

$-0.586690 \mathrm{D}+00 \quad-0.579214 \mathrm{D}+00 \quad-0.729424 \mathrm{D}+00 \quad-0.715277 \mathrm{D}+00 \quad-0.866030 \mathrm{D}+00 \quad-0.938560 \mathrm{D}+00 \quad-0.641560 \mathrm{D}+00$ $-0.787835 \mathrm{D}+00-0.411694 \mathrm{D}+00 \quad-0.239347 \mathrm{D}+00 \quad-0.637287 \mathrm{D}+00 \quad-0.971247 \mathrm{D}+00 \quad-0.347305 \mathrm{D}+00$

$-0.211988 \mathrm{D}+01 \quad-0.285480 \mathrm{D}+01 \quad-0.273180 \mathrm{D}+01 \quad-0.722856 \mathrm{D}+00 \quad-0.111011 \mathrm{D}+01 \quad-0.690442 \mathrm{D}+00 \quad-0.737893 \mathrm{D}+00$ $\begin{array}{llllll}-0.800005 \mathrm{D}+00 & -0.206086 \mathrm{D}-01 & -0.346838 \mathrm{D}+00 & -0.357376 \mathrm{D}+00 & 0.397890 \mathrm{D}+00 & -0.248841 \mathrm{D}+00\end{array}$

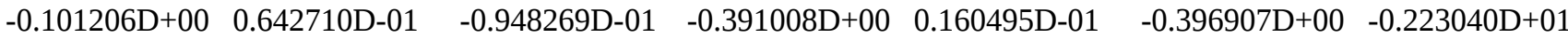
$-0.478491 \mathrm{D}+00$

$-0.339456 \mathrm{D}+01 \quad-0.342022 \mathrm{D}+01 \quad-0.294972 \mathrm{D}+01 \quad-0.299970 \mathrm{D}+01 \quad-0.212722 \mathrm{D}+01 \quad-0.233429 \mathrm{D}+01-0.205068 \mathrm{D}+01$ $-0.267254 \mathrm{D}+01 \quad-0.815219 \mathrm{D}+00 \quad-0.966681 \mathrm{D}-01 \quad-0.117278 \mathrm{D}+01 \quad-0.767530 \mathrm{D}+00 \quad-0.791597 \mathrm{D}+00$

$-0.644509 \mathrm{D}+00 \quad-0.910943 \mathrm{D}+00 \quad-0.521966 \mathrm{D}+00 \quad-0.294282 \mathrm{D}+00 \quad-0.120319 \mathrm{D}+01 \quad-0.511998 \mathrm{D}+00 \quad-0.144136 \mathrm{D}+00$ $\begin{array}{llllll}-0.392824 \mathrm{D}+00 & -0.799597 \mathrm{D}-01 & -0.168429 \mathrm{D}+00 & 0.624064 \mathrm{D}-01 & 0.995319 \mathrm{D}-02 & 0.123927 \mathrm{D}+00\end{array}$

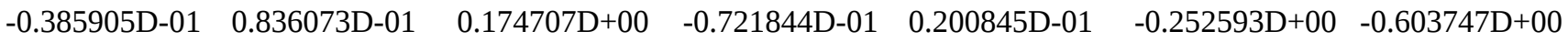
$-0.566918 \mathrm{D}+00-0.217690 \mathrm{D}+01$

$-0.346755 \mathrm{D}+01 \quad-0.349488 \mathrm{D}+01 \quad-0.298399 \mathrm{D}+01 \quad-0.303770 \mathrm{D}+01 \quad-0.210237 \mathrm{D}+01 \quad-0.230992 \mathrm{D}+01 \quad-0.204654 \mathrm{D}+01$ $-0.268595 \mathrm{D}+01 \quad-0.764693 \mathrm{D}+00 \quad-0.257789 \mathrm{D}-01 \quad-0.114613 \mathrm{D}+01 \quad-0.749119 \mathrm{D}+00 \quad-0.788363 \mathrm{D}+00$

$-0.724257 \mathrm{D}+00 \quad-0.931760 \mathrm{D}+00 \quad-0.522724 \mathrm{D}+00 \quad-0.234624 \mathrm{D}+01 \quad-0.434742 \mathrm{D}+00 \quad-0.543129 \mathrm{D}+00 \quad-0.139553 \mathrm{D}+00$ $\begin{array}{llllll}0.104053 \mathrm{D}+01 & -0.825178 \mathrm{D}-01 & -0.147825 \mathrm{D}+00 & -0.915498 \mathrm{D}-01 & -0.400759 \mathrm{D}-01 & 0.171204 \mathrm{D}+00\end{array}$ $\begin{array}{llllll}0.683224 \mathrm{D}-01 & 0.426570 \mathrm{D}-01 & 0.212223 \mathrm{D}+00 & -0.137990 \mathrm{D}+00 & -0.817031 \mathrm{D}-01 & -0.242046 \mathrm{D}+00\end{array}$

$-0.624792 \mathrm{D}+00 \quad-0.584625 \mathrm{D}+00 \quad-0.864665 \mathrm{D}+01 \quad-0.212179 \mathrm{D}+01$

$-0.197969 \mathrm{D}+01 \quad-0.196529 \mathrm{D}+01 \quad-0.230332 \mathrm{D}+01 \quad-0.228925 \mathrm{D}+01 \quad-0.258114 \mathrm{D}+01 \quad-0.284628 \mathrm{D}+01 \quad-0.193175 \mathrm{D}+01$

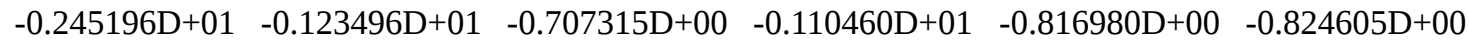

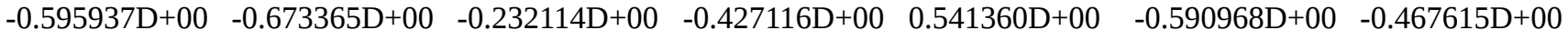
$\begin{array}{llllll}-0.130009 D+00 & -0.263925 \mathrm{D}+00 & -0.165795 \mathrm{D}+00 & -0.244493 \mathrm{D}+00 & 0.833984 \mathrm{D}-01 & 0.457027 \mathrm{D}+00\end{array}$ $\begin{array}{llllll}0.184163 \mathrm{D}+00 & 0.539951 \mathrm{D}-01 & 0.298445 \mathrm{D}+00 & -0.203616 \mathrm{D}+00 & -0.137910 \mathrm{D}+00 & -0.323205 \mathrm{D}+00\end{array}$ 
0.217903D-02 $\quad 0.481064 \mathrm{D}-01 \quad-0.443597 \mathrm{D}+01 \quad-0.430250 \mathrm{D}+01 \quad-0.864088 \mathrm{D}+00$

$-0.227356 \mathrm{D}+01 \quad-0.226403 \mathrm{D}+01 \quad-0.253822 \mathrm{D}+01 \quad-0.253388 \mathrm{D}+01 \quad-0.264550 \mathrm{D}+01 \quad-0.299482 \mathrm{D}+01 \quad-0.191478 \mathrm{D}+01$ $-0.265004 \mathrm{D}+01 \quad-0.814892 \mathrm{D}+00 \quad-0.106757 \mathrm{D}-01 \quad-0.119341 \mathrm{D}+01 \quad-0.699666 \mathrm{D}+00 \quad-0.796018 \mathrm{D}+00$

$-0.629821 \mathrm{D}+00 \quad-0.700125 \mathrm{D}+00 \quad-0.910318 \mathrm{D}-01 \quad-0.113642 \mathrm{D}+01 \quad-0.140846 \mathrm{D}+00 \quad-0.110539 \mathrm{D}+01 \quad-0.313227 \mathrm{D}+00$ $\begin{array}{llllll}0.128537 \mathrm{D}+00 & 0.582542 \mathrm{D}-01 & -0.121596 \mathrm{D}+00 & -0.904295 \mathrm{D}-01 & -0.219662 \mathrm{D}-01 & 0.276263 \mathrm{D}+00\end{array}$ $0.140751 \mathrm{D}+00 \quad 0.756832 \mathrm{D}-01 \quad 0.176240 \mathrm{D}+00 \quad-0.216085 \mathrm{D}+00 \quad-0.176290 \mathrm{D}+00 \quad-0.363292 \mathrm{D}+00$

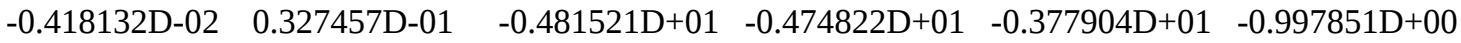

$-0.525729 \mathrm{D}+00 \quad-0.520256 \mathrm{D}+00 \quad-0.505207 \mathrm{D}+00 \quad-0.481117 \mathrm{D}+00 \quad-0.954455 \mathrm{D}+00 \quad-0.416718 \mathrm{D}+00 \quad-0.167982 \mathrm{D}+01$ $\begin{array}{llllll}-0.429309 D+00 & -0.384006 \mathrm{D}+01 & -0.600816 \mathrm{D}+01 & -0.106797 \mathrm{D}+01 & -0.139161 \mathrm{D}+01 & -0.100924 \mathrm{D}+01\end{array}$

$-0.841151 \mathrm{D}+00 \quad-0.514031 \mathrm{D}+00 \quad-0.763339 \mathrm{D}+00 \quad-0.608338 \mathrm{D}+00 \quad 0.685764 \mathrm{D}-01 \quad-0.699482 \mathrm{D}+00 \quad-0.700068 \mathrm{D}+00$ $\begin{array}{llllll}-0.287894 \mathrm{D}+00 & -0.133033 \mathrm{D}+00 & 0.478126 \mathrm{D}+00 & -0.931660 \mathrm{D}+00 & 0.105498 \mathrm{D}+01 & -0.250157 \mathrm{D}+00\end{array}$ $0.366371 \mathrm{D}+00 \quad 0.682587 \mathrm{D}+00 \quad 0.386293 \mathrm{D}-01 \quad-0.138354 \mathrm{D}-01 \quad 0.439223 \mathrm{D}-01 \quad-0.112834 \mathrm{D}+00$

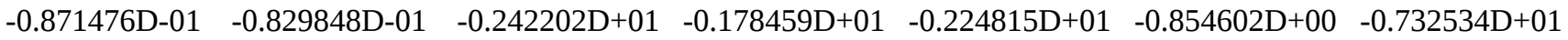

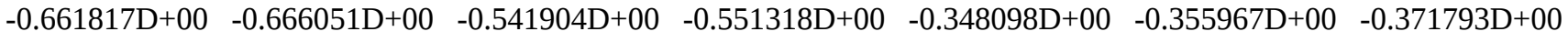
$-0.416836 \mathrm{D}+00 \quad-0.218475 \mathrm{D}+00 \quad-0.141814 \mathrm{D}+00 \quad-0.146010 \mathrm{D}+01 \quad-0.252543 \mathrm{D}+01 \quad-0.896720 \mathrm{D}+00$

$-0.104732 \mathrm{D}+01 \quad-0.205891 \mathrm{D}+00 \quad-0.724206 \mathrm{D}+00 \quad-0.730845 \mathrm{D}+00 \quad-0.828692 \mathrm{D}+00 \quad-0.115851 \mathrm{D}+01 \quad-0.677153 \mathrm{D}+00$ $-0.229568 \mathrm{D}+00 \quad-0.762265 \mathrm{D}+00 \quad-0.186733 \mathrm{D}+00 \quad-0.783621 \mathrm{D}+00 \quad-0.255202 \mathrm{D}-01 \quad-0.184381 \mathrm{D}+00$

$-0.757436 \mathrm{D}-01 \quad-0.203280 \mathrm{D}+00 \quad-0.856176 \mathrm{D}-02 \quad-0.862671 \mathrm{D}-02 \quad-0.102832 \mathrm{D}+00 \quad-0.103351 \mathrm{D}+00 \quad-0.542609 \mathrm{D}+00$ $\begin{array}{llllll}-0.527015 \mathrm{D}+00 & -0.266901 \mathrm{D}+00 & -0.249226 \mathrm{D}+00 & -0.917247 \mathrm{D}-01 & -0.905786 \mathrm{D}-01 & -0.670205 \mathrm{D}-01\end{array}$ $0.112931 \mathrm{D}-01$

$-0.500473 \mathrm{D}+00 \quad-0.496375 \mathrm{D}+00 \quad-0.493458 \mathrm{D}+00 \quad-0.493271 \mathrm{D}+00 \quad-0.548923 \mathrm{D}+00 \quad-0.542930 \mathrm{D}+00 \quad-0.501734 \mathrm{D}+00$ $-0.478718 \mathrm{D}+00 \quad-0.520708 \mathrm{D}+00-0.539249 \mathrm{D}+00 \quad-0.105307 \mathrm{D}+01-0.156663 \mathrm{D}+01-0.101888 \mathrm{D}+01$

$-0.124620 \mathrm{D}+01-0.104175 \mathrm{D}+01 \quad-0.675325 \mathrm{D}+00 \quad-0.333359 \mathrm{D}+00 \quad-0.421345 \mathrm{D}+00 \quad-0.129571 \mathrm{D}+01 \quad-0.942360 \mathrm{D}+00$ $\begin{array}{llllll}-0.307552 \mathrm{D}+00 & -0.863047 \mathrm{D}+00 & -0.204836 \mathrm{D}+00 & -0.246935 \mathrm{D}+00 & -0.187597 \mathrm{D}+00 & -0.979338 \mathrm{D}-01\end{array}$ $\begin{array}{llllll}0.636867 \mathrm{D}-01 & -0.668976 \mathrm{D}-01 & 0.205938 \mathrm{D}+00 & -0.134173 \mathrm{D}+00 & 0.110282 \mathrm{D}-01 & -0.347166 \mathrm{D}+00\end{array}$

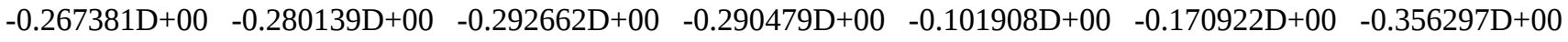
$0.183632 \mathrm{D}+00 \quad 0.240088 \mathrm{D}-01$

$-0.634976 \mathrm{D}+00 \quad-0.636388 \mathrm{D}+00 \quad-0.483987 \mathrm{D}+00 \quad-0.452903 \mathrm{D}+00 \quad-0.900176 \mathrm{D}+00 \quad-0.160304 \mathrm{D}+00 \quad-0.208529 \mathrm{D}+01$ $-0.328196 \mathrm{D}+00 \quad-0.503822 \mathrm{D}+01 \quad-0.624864 \mathrm{D}+01 \quad-0.485851 \mathrm{D}+00 \quad-0.110673 \mathrm{D}+01 \quad-0.138001 \mathrm{D}+01$

$-0.715858 \mathrm{D}+00 \quad-0.539324 \mathrm{D}+00 \quad-0.371268 \mathrm{D}+00 \quad-0.104543 \mathrm{D}+01 \quad-0.178361 \mathrm{D}+00 \quad-0.438794 \mathrm{D}+00 \quad-0.377088 \mathrm{D}+00$ $\begin{array}{llllll}-0.122667 \mathrm{D}+00 & 0.100308 \mathrm{D}+00 & -0.899419 \mathrm{D}+00 & -0.888902 \mathrm{D}+00 & 0.998489 \mathrm{D}+00 & 0.106070 \mathrm{D}-01\end{array}$ $\begin{array}{llllll}0.467233 \mathrm{D}+00 & 0.550226 \mathrm{D}+00 & 0.605053 \mathrm{D}-02 & -0.278497 \mathrm{D}+00 & -0.322820 \mathrm{D}-01 & 0.370913 \mathrm{D}-03\end{array}$

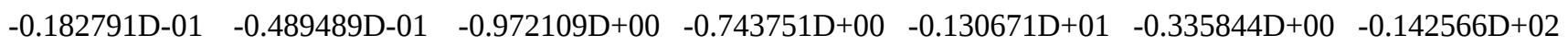
$-0.171443 \mathrm{D}+00 \quad-0.296965 \mathrm{D}+00 \quad-0.294515 \mathrm{D}+01$

$-0.178265 \mathrm{D}+01 \quad-0.177733 \mathrm{D}+01 \quad-0.194396 \mathrm{D}+01 \quad-0.194175 \mathrm{D}+01 \quad-0.201089 \mathrm{D}+01 \quad-0.226239 \mathrm{D}+01 \quad-0.147450 \mathrm{D}+01$ $\begin{array}{llllll}-0.201409 \mathrm{D}+01 & -0.685605 \mathrm{D}+00 & -0.949196 \mathrm{D}-01 & -0.673106 \mathrm{D}+00 & -0.673464 \mathrm{D}+00 & 0.228273 \mathrm{D}+00\end{array}$

$-0.473990 \mathrm{D}+00 \quad-0.636018 \mathrm{D}+00 \quad-0.544962 \mathrm{D}+00 \quad-0.530308 \mathrm{D}+00 \quad-0.202545 \mathrm{D}+00 \quad-0.693783 \mathrm{D}-01 \quad-0.514320 \mathrm{D}+00$ $\begin{array}{llllll}0.608019 D-01 & -0.913773 D-01 & 0.376076 \mathrm{D}-01 & -0.353902 \mathrm{D}+00 & -0.895445 \mathrm{D}-01 & 0.195271 \mathrm{D}+00\end{array}$ $\begin{array}{llllll}0.120953 \mathrm{D}+00 & 0.323793 \mathrm{D}+00 & 0.618378 \mathrm{D}+00 & 0.446934 \mathrm{D}+00 & 0.223629 \mathrm{D}+00 & -0.157537 \mathrm{D}+00\end{array}$

$-0.161566 \mathrm{D}+00 \quad-0.217239 \mathrm{D}+00 \quad-0.466663 \mathrm{D}+01-0.454439 \mathrm{D}+01 \quad-0.356213 \mathrm{D}+01 \quad-0.369558 \mathrm{D}+01 \quad-0.360592 \mathrm{D}+01$ $-0.272492 \mathrm{D}+00 \quad-0.290069 \mathrm{D}+00 \quad-0.557655 \mathrm{D}+00 \quad-0.135469 \mathrm{D}+01$

$-0.141391 \mathrm{D}+01-0.140523 \mathrm{D}+01 \quad-0.156742 \mathrm{D}+01-0.155024 \mathrm{D}+01 \quad-0.187557 \mathrm{D}+01 \quad-0.184875 \mathrm{D}+01-0.177981 \mathrm{D}+01$ $-0.163575 \mathrm{D}+01 \quad-0.215268 \mathrm{D}+01-0.209587 \mathrm{D}+01 \quad-0.611111 \mathrm{D}+00 \quad-0.686547 \mathrm{D}+00 \quad-0.586159 \mathrm{D}+00$

$-0.524806 \mathrm{D}+00 \quad-0.655757 \mathrm{D}+00 \quad-0.457933 \mathrm{D}+00 \quad-0.332116 \mathrm{D}+00 \quad-0.123783 \mathrm{D}+00 \quad-0.128396 \mathrm{D}+01 \quad-0.208003 \mathrm{D}+00$ $\begin{array}{llllll}0.649831 \mathrm{D}-01 & -0.851694 \mathrm{D}-01 & -0.271017 \mathrm{D}+00 & -0.351504 \mathrm{D}+00 & 0.826395 \mathrm{D}-01 & 0.189439 \mathrm{D}+00\end{array}$ $0.602542 \mathrm{D}+00 \quad 0.210666 \mathrm{D}+00 \quad 0.526256 \mathrm{D}+00 \quad 0.288495 \mathrm{D}+00 \quad 0.141091 \mathrm{D}+00 \quad 0.540401 \mathrm{D}-01$

$-0.248244 \mathrm{D}+00 \quad-0.131821 \mathrm{D}+00 \quad-0.326465 \mathrm{D}+01 \quad-0.315027 \mathrm{D}+01 \quad-0.318258 \mathrm{D}+01 \quad-0.278485 \mathrm{D}+01 \quad-0.551854 \mathrm{D}+01$

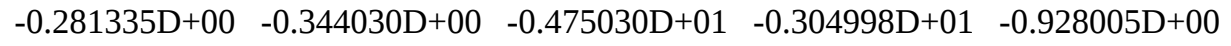

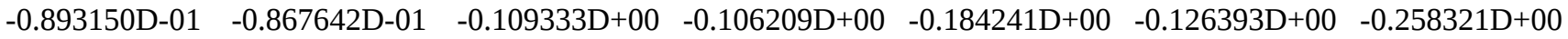

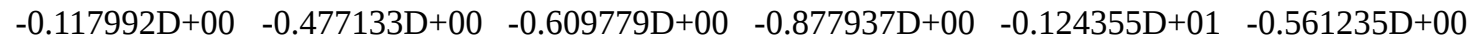


$-0.420636 \mathrm{D}+01 \quad-0.686873 \mathrm{D}+00 \quad-0.139809 \mathrm{D}+01 \quad-0.538563 \mathrm{D}+00 \quad-0.418175 \mathrm{D}+00 \quad-0.150022 \mathrm{D}+01 \quad-0.205631 \mathrm{D}+01$

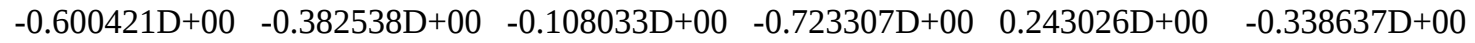

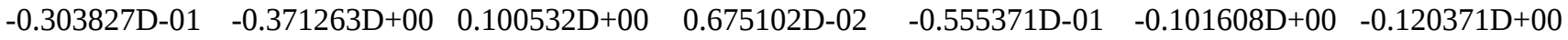
$\begin{array}{llllll}-0.209263 \mathrm{D}-01 & -0.847548 \mathrm{D}-01 & -0.875464 \mathrm{D}-01 & -0.145835 \mathrm{D}+00 & -0.167833 \mathrm{D}+00 & -0.186410 \mathrm{D}-01\end{array}$

$\begin{array}{llllll}-0.243620 \mathrm{D}+00 & 0.497926 \mathrm{D}+00 & 0.265539 \mathrm{D}-01 & -0.289489 \mathrm{D}+00 & -0.381289 \mathrm{D}-01 & 0.756151 \mathrm{D}+00\end{array}$

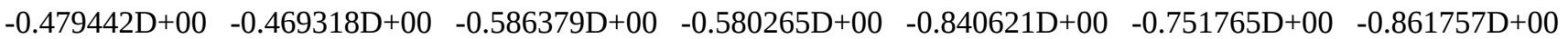
$-0.621390 \mathrm{D}+00 \quad-0.128630 \mathrm{D}+01 \quad-0.147458 \mathrm{D}+01 \quad-0.621939 \mathrm{D}+00 \quad-0.894469 \mathrm{D}+00 \quad-0.628393 \mathrm{D}+00$

$-0.106728 \mathrm{D}+01 \quad-0.456425 \mathrm{D}+00 \quad-0.380016 \mathrm{D}+00 \quad-0.373606 \mathrm{D}+00 \quad-0.320483 \mathrm{D}+00 \quad-0.125198 \mathrm{D}+01 \quad-0.152497 \mathrm{D}+01$ $\begin{array}{llllll}0.811229 \mathrm{D}-01 & -0.648578 \mathrm{D}+00 & 0.111713 \mathrm{D}+01 & -0.473089 \mathrm{D}+00 & -0.496683 \mathrm{D}-01 & -0.157478 \mathrm{D}+00\end{array}$

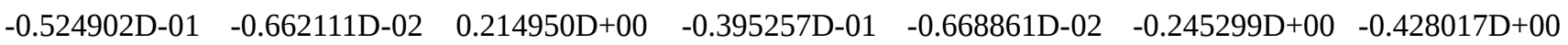
$-0.205473 \mathrm{D}+00 \quad-0.247491 \mathrm{D}+01-0.211491 \mathrm{D}+01 \quad-0.117004 \mathrm{D}+01-0.979887 \mathrm{D}+00 \quad-0.871564 \mathrm{D}+01$

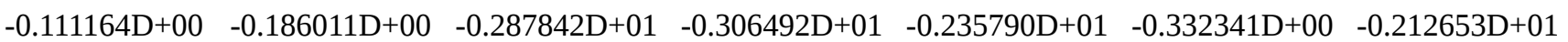

$-0.220978 \mathrm{D}+01 \quad-0.222615 \mathrm{D}+01 \quad-0.194140 \mathrm{D}+01 \quad-0.197142 \mathrm{D}+01 \quad-0.139278 \mathrm{D}+01 \quad-0.152819 \mathrm{D}+01 \quad-0.134469 \mathrm{D}+01$

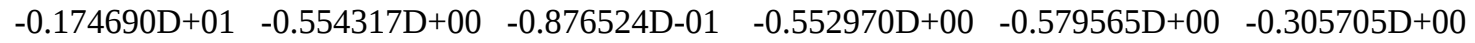

$-0.422809 \mathrm{D}+00 \quad-0.394307 \mathrm{D}+00 \quad-0.101334 \mathrm{D}+00 \quad-0.309609 \mathrm{D}+00 \quad 0.260307 \mathrm{D}-01 \quad-0.276348 \mathrm{D}+00 \quad-0.124290 \mathrm{D}+00$ $\begin{array}{llllll}0.166608 \mathrm{D}+00 & 0.166017 \mathrm{D}+00 & 0.185887 \mathrm{D}+00 & 0.425975 \mathrm{D}+00 & -0.142686 \mathrm{D}-01 & -0.107087 \mathrm{D}+00\end{array}$ $\begin{array}{llllll}0.219244 \mathrm{D}+00 & 0.342205 \mathrm{D}+01 & 0.113285 \mathrm{D}+01 & 0.520657 \mathrm{D}+00 & -0.781096 \mathrm{D}+00 & 0.431384 \mathrm{D}+00\end{array}$ 0.845009D-01 $\quad 0.592882 \mathrm{D}-01 \quad-0.286548 \mathrm{D}+01 \quad-0.294168 \mathrm{D}+01 \quad-0.210123 \mathrm{D}+01 \quad-0.225227 \mathrm{D}+01$

$-0.100693 \mathrm{D}+01 \quad-0.418031 \mathrm{D}+00 \quad 0.223339 \mathrm{D}+00 \quad-0.441715 \mathrm{D}+00 \quad-0.267942 \mathrm{D}+01 \quad-0.163956 \mathrm{D}+01 \quad-0.172035 \mathrm{D}+00$ $-0.643254 \mathrm{D}+00-0.442933 \mathrm{D}+00$

$-0.604764 \mathrm{D}+00 \quad-0.601439 \mathrm{D}+00 \quad-0.703070 \mathrm{D}+00 \quad-0.703823 \mathrm{D}+00 \quad-0.813794 \mathrm{D}+00 \quad-0.877456 \mathrm{D}+00 \quad-0.632330 \mathrm{D}+00$

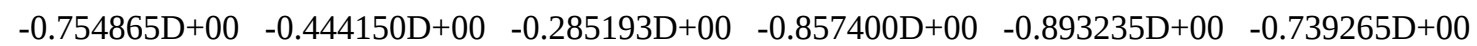

$-0.112550 \mathrm{D}+01 \quad-0.804831 \mathrm{D}+00 \quad-0.628479 \mathrm{D}+00 \quad-0.493467 \mathrm{D}+00 \quad-0.479698 \mathrm{D}+00 \quad-0.139653 \mathrm{D}+01 \quad-0.143912 \mathrm{D}+01$ $\begin{array}{llllll}-0.650209 \mathrm{D}-01 & -0.729796 \mathrm{D}+00 & 0.150886 \mathrm{D}+00 & 0.537005 \mathrm{D}+00 & 0.169434 \mathrm{D}+01 & 0.125733 \mathrm{D}+01\end{array}$

$-0.256867 \mathrm{D}+00 \quad-0.291743 \mathrm{D}+00 \quad 0.263253 \mathrm{D}+00 \quad-0.838449 \mathrm{D}-02 \quad 0.178463 \mathrm{D}-01 \quad-0.781311 \mathrm{D}+00 \quad-0.419307 \mathrm{D}+00$ $-0.160336 \mathrm{D}+01 \quad-0.302582 \mathrm{D}+01 \quad-0.282381 \mathrm{D}+01 \quad-0.132646 \mathrm{D}+01-0.136083 \mathrm{D}+01 \quad-0.524876 \mathrm{D}+01$

$-0.138602 \mathrm{D}+00 \quad-0.228611 \mathrm{D}+00 \quad-0.651998 \mathrm{D}+00 \quad-0.415938 \mathrm{D}+01 \quad-0.164636 \mathrm{D}+01 \quad-0.508391 \mathrm{D}+00 \quad-0.700823 \mathrm{D}+01$ $-0.101427 \mathrm{D}+01-0.220737 \mathrm{D}+01$

$-0.142528 \mathrm{D}+00 \quad-0.140900 \mathrm{D}+00 \quad-0.185612 \mathrm{D}+00 \quad-0.184718 \mathrm{D}+00 \quad-0.221440 \mathrm{D}+00 \quad-0.206479 \mathrm{D}+00 \quad-0.244366 \mathrm{D}+00$ $\begin{array}{llllll}-0.191721 \mathrm{D}+00 & -0.334433 \mathrm{D}+00 & -0.388982 \mathrm{D}+00 & -0.392466 \mathrm{D}+00 & -0.183052 \mathrm{D}+01 & -0.553435 \mathrm{D}+00\end{array}$ $0.170596 \mathrm{D}+01 \quad-0.537662 \mathrm{D}+00 \quad-0.133739 \mathrm{D}+01 \quad-0.167801 \mathrm{D}-01 \quad-0.323969 \mathrm{D}+00 \quad-0.268602 \mathrm{D}+01$

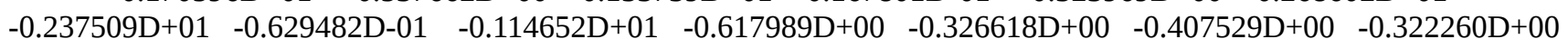
$\begin{array}{llllll}-0.840936 \mathrm{D}-01 & -0.960122 \mathrm{D}-01 & 0.111426 \mathrm{D}+00 & -0.115837 \mathrm{D}+00 & -0.287894 \mathrm{D}+00 & -0.119815 \mathrm{D}+00\end{array}$

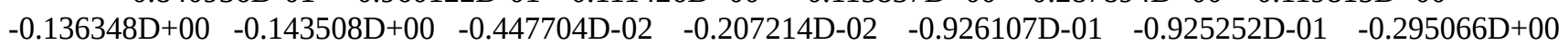
$0.111398 \mathrm{D}+01 \quad-0.272716 \mathrm{D}+00 \quad-0.330874 \mathrm{D}+00 \quad-0.223987 \mathrm{D}+00 \quad-0.115964 \mathrm{D}+00 \quad 0.678600 \mathrm{D}-01$

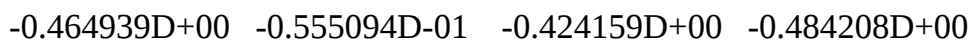

$-0.197184 \mathrm{D}+01-0.198236 \mathrm{D}+01 \quad-0.178538 \mathrm{D}+01 \quad-0.180533 \mathrm{D}+01 \quad-0.142468 \mathrm{D}+01 \quad-0.149651 \mathrm{D}+01 \quad-0.141631 \mathrm{D}+01$ $\begin{array}{llllll}-0.164346 \mathrm{D}+01 & -0.954554 \mathrm{D}+00 & -0.628832 \mathrm{D}+00 & -0.116299 \mathrm{D}+01 & -0.412658 \mathrm{D}+00 & 0.319217 \mathrm{D}+00\end{array}$

$-0.453878 \mathrm{D}+00 \quad-0.362483 \mathrm{D}+00 \quad-0.257932 \mathrm{D}-02 \quad-0.212277 \mathrm{D}+00 \quad-0.340812 \mathrm{D}-01 \quad-0.441014 \mathrm{D}+00 \quad-0.330385 \mathrm{D}+00$ $\begin{array}{llllll}0.209365 \mathrm{D}+00 & 0.131377 \mathrm{D}+00 & -0.158475 \mathrm{D}+01 & 0.181345 \mathrm{D}+00 & 0.157431 \mathrm{D}+00 & 0.255650 \mathrm{D}+00\end{array}$ $\begin{array}{llllll}0.220227 \mathrm{D}+00 & 0.616463 \mathrm{D}+00 & 0.124935 \mathrm{D}+01 & 0.682369 \mathrm{D}+00 & 0.677536 \mathrm{D}+00 & 0.187568 \mathrm{D}+00\end{array}$ $\begin{array}{llllll}0.935640 \mathrm{D}-01 & 0.135300 \mathrm{D}+00 & -0.262707 \mathrm{D}+01 & -0.257105 \mathrm{D}+01 & -0.224224 \mathrm{D}+01 & -0.224388 \mathrm{D}+01\end{array}$

$-0.143634 \mathrm{D}+01-0.426568 \mathrm{D}+00 \quad-0.518044 \mathrm{D}+00 \quad-0.167149 \mathrm{D}+01 \quad-0.294944 \mathrm{D}+01 \quad-0.219168 \mathrm{D}+01 \quad-0.837915 \mathrm{D}-01$ $-0.105503 \mathrm{D}+01-0.157286 \mathrm{D}+01 \quad-0.103916 \mathrm{D}+01 \quad-0.761916 \mathrm{D}-01 \quad-0.425925 \mathrm{D}+00$

$-0.268494 \mathrm{D}+00 \quad-0.271277 \mathrm{D}+00 \quad-0.201294 \mathrm{D}+00 \quad-0.210455 \mathrm{D}+00 \quad-0.157518 \mathrm{D}+00 \quad-0.147811 \mathrm{D}+00 \quad-0.200822 \mathrm{D}+00$ $-0.200639 \mathrm{D}+00-0.150987 \mathrm{D}+00-0.160011 \mathrm{D}+00 \quad-0.935718 \mathrm{D}+00-0.156281 \mathrm{D}+01 \quad-0.502196 \mathrm{D}+00$

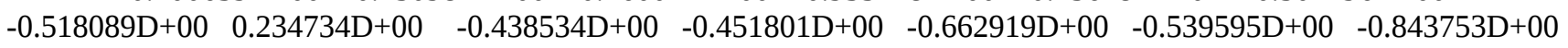

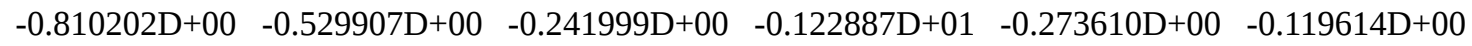

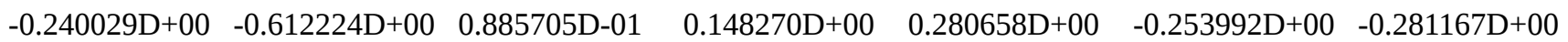
$\begin{array}{llllll}-0.452952 \mathrm{D}+00 & -0.288504 \mathrm{D}-01 & 0.209917 \mathrm{D}-02 & -0.248840 \mathrm{D}-01 & -0.160078 \mathrm{D}-01 & -0.258939 \mathrm{D}+00\end{array}$

$-0.104780 \mathrm{D}+01 \quad-0.113592 \mathrm{D}+00 \quad-0.278260 \mathrm{D}+00 \quad-0.184130 \mathrm{D}+00 \quad-0.175972 \mathrm{D}+00 \quad-0.666814 \mathrm{D}-01 \quad 0.116546 \mathrm{D}+00$ $\begin{array}{lllll}-0.286228 \mathrm{D}-01 & -0.165805 \mathrm{D}+00 & -0.223003 \mathrm{D}+00 & -0.254670 \mathrm{D}-01 & 0.158492 \mathrm{D}+00\end{array}$ 
$-0.113698 \mathrm{D}+00 \quad-0.114204 \mathrm{D}+00 \quad-0.163722 \mathrm{D}+00 \quad-0.161905 \mathrm{D}+00 \quad-0.158786 \mathrm{D}+00 \quad-0.153623 \mathrm{D}+00-0.171056 \mathrm{D}+00$ $-0.166691 \mathrm{D}+00 \quad-0.167748 \mathrm{D}+00 \quad-0.150163 \mathrm{D}+00 \quad-0.662079 \mathrm{D}+00 \quad-0.103626 \mathrm{D}+01 \quad-0.895991 \mathrm{D}+00$

$-0.693805 \mathrm{D}+00 \quad-0.886091 \mathrm{D}+00 \quad-0.585672 \mathrm{D}+00 \quad-0.477175 \mathrm{D}+00 \quad-0.533441 \mathrm{D}+00 \quad-0.343314 \mathrm{D}+00 \quad-0.760168 \mathrm{D}+00$

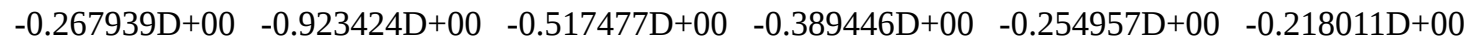
$0.356862 \mathrm{D}+00 \quad-0.189474 \mathrm{D}+00 \quad 0.573118 \mathrm{D}-01 \quad-0.455472 \mathrm{D}-01 \quad-0.228221 \mathrm{D}+00 \quad-0.169560 \mathrm{D}+00$

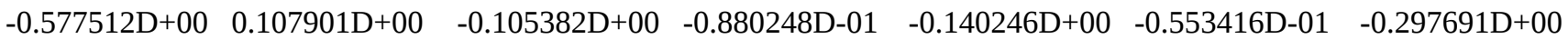

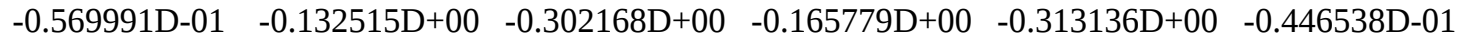

$\begin{array}{lllllll}-0.243448 \mathrm{D}+00 & -0.469969 \mathrm{D}-01 & -0.209970 \mathrm{D}+00 & -0.454188 \mathrm{D}+00 & 0.677312 \mathrm{D}-01 & -0.917769 \mathrm{D}-01 & 0.616223 \mathrm{D}-01\end{array}$

$-0.162269 \mathrm{D}+00 \quad-0.162795 \mathrm{D}+00 \quad-0.186946 \mathrm{D}+00 \quad-0.191415 \mathrm{D}+00 \quad-0.936164 \mathrm{D}-01 \quad-0.101701 \mathrm{D}+00 \quad-0.123736 \mathrm{D}+00$ $\begin{array}{llllll}-0.162064 \mathrm{D}+00 & -0.398387 \mathrm{D}-01 & 0.223989 \mathrm{D}-02 & -0.125488 \mathrm{D}+01 & -0.897900 \mathrm{D}+00 & -0.574236 \mathrm{D}+00\end{array}$

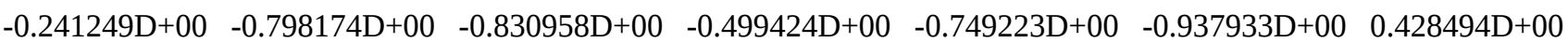

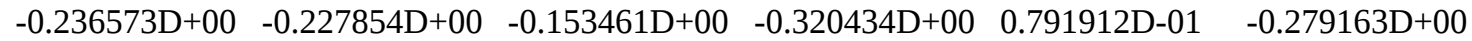

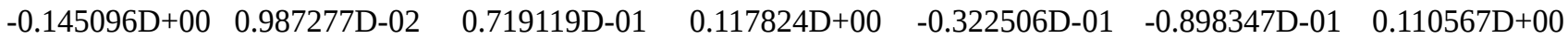
0.579131D-01 -0.748265D-01 $-0.576724 \mathrm{D}-01 \quad-0.727252 \mathrm{D}-01 \quad-0.416037 \mathrm{D}-01 \quad-0.724884 \mathrm{D}-01$

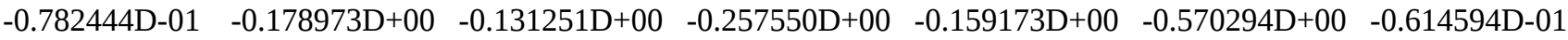
$\begin{array}{llllll}0.493090 \mathrm{D}-01 & -0.222856 \mathrm{D}+00 & -0.102297 \mathrm{D}+01 & -0.983631 \mathrm{D}-02 & -0.283766 \mathrm{D}-01 & 0.193858 \mathrm{D}+00\end{array}$ $0.655807 \mathrm{D}-01$

$-0.551275 \mathrm{D}+00 \quad-0.554190 \mathrm{D}+00 \quad-0.495300 \mathrm{D}+00 \quad-0.505543 \mathrm{D}+00 \quad-0.403471 \mathrm{D}+00 \quad-0.416678 \mathrm{D}+00 \quad-0.421134 \mathrm{D}+00$

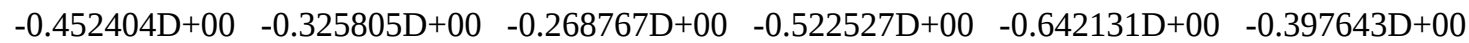

$-0.649916 \mathrm{D}+00 \quad-0.838752 \mathrm{D}+00 \quad-0.739542 \mathrm{D}+00 \quad-0.765909 \mathrm{D}+00 \quad-0.469368 \mathrm{D}+00 \quad-0.191608 \mathrm{D}+01 \quad-0.506857 \mathrm{D}+00$ $\begin{array}{llllll}-0.265996 \mathrm{D}+00 & -0.658640 \mathrm{D}+00 & -0.188043 \mathrm{D}+01 & -0.313270 \mathrm{D}+00 & -0.185643 \mathrm{D}+00 & -0.136443 \mathrm{D}+00\end{array}$

$-0.263465 \mathrm{D}+00 \quad-0.229134 \mathrm{D}+00 \quad-0.198561 \mathrm{D}+00 \quad-0.136711 \mathrm{D}+00 \quad 0.153195 \mathrm{D}+00 \quad 0.399488 \mathrm{D}+00 \quad-0.861619 \mathrm{D}+00$

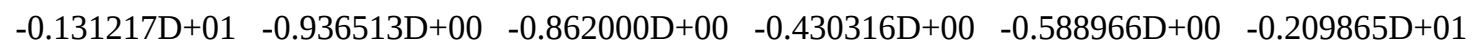
$\begin{array}{llllll}0.152853 \mathrm{D}+00 & -0.359358 \mathrm{D}+00 & -0.361634 \mathrm{D}+00 & -0.868017 \mathrm{D}+00 & 0.375608 \mathrm{D}+00 & -0.149018 \mathrm{D}+00\end{array}$

$-0.123116 \mathrm{D}+01 \quad-0.497772 \mathrm{D}+00 \quad-0.134921 \mathrm{D}+01 \quad-0.261760 \mathrm{D}-01 \quad 0.108777 \mathrm{D}+01 \quad-0.116277 \mathrm{D}+00 \quad-0.231050 \mathrm{D}-01$ $-0.111017 \mathrm{D}+00 \quad-0.251579 \mathrm{D}+00$

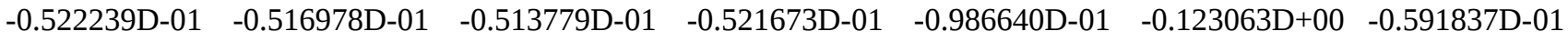

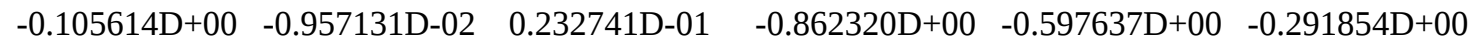

$-0.104937 \mathrm{D}+01-0.788341 \mathrm{D}+00 \quad-0.498504 \mathrm{D}+00 \quad-0.489053 \mathrm{D}+00 \quad-0.280488 \mathrm{D}+00 \quad-0.754374 \mathrm{D}+00 \quad-0.876148 \mathrm{D}+00$ $\begin{array}{llllll}-0.237740 \mathrm{D}+00 & -0.544487 \mathrm{D}+00 & -0.144455 \mathrm{D}+00 & -0.189926 \mathrm{D}+00 & 0.190588 \mathrm{D}+00 & -0.350170 \mathrm{D}-01\end{array}$ $\begin{array}{llllll}0.415595 \mathrm{D}+00 & -0.562540 \mathrm{D}+00 & 0.415624 \mathrm{D}+00 & 0.252618 \mathrm{D}+00 & 0.470751 \mathrm{D}+00 & 0.119766 \mathrm{D}+00\end{array}$

$-0.488727 \mathrm{D}+00 \quad-0.224361 \mathrm{D}+00 \quad-0.261590 \mathrm{D}-01 \quad-0.225352 \mathrm{D}-01 \quad-0.263493 \mathrm{D}-01 \quad-0.186380 \mathrm{D}-01 \quad 0.672050 \mathrm{D}-02$ $\begin{array}{llllll}-0.248965 \mathrm{D}+00 & 0.160878 \mathrm{D}+00 & -0.143444 \mathrm{D}+00 & -0.820673 \mathrm{D}-01 & -0.557667 \mathrm{D}-01 & 0.730282 \mathrm{D}-01\end{array}$ $\begin{array}{llllll}0.110347 \mathrm{D}+00 & -0.132152 \mathrm{D}-01 & -0.382518 \mathrm{D}-01 & -0.245973 \mathrm{D}+00 & 0.800630 \mathrm{D}-01 & 0.663928 \mathrm{D}+00\end{array}$ $-0.217910 \mathrm{D}+00 \quad 0.393401 \mathrm{D}-01 \quad-0.659238 \mathrm{D}-01 \quad 0.706581 \mathrm{D}-01$

$-0.464340 \mathrm{D}+00 \quad-0.467868 \mathrm{D}+00 \quad-0.383967 \mathrm{D}+00 \quad-0.392036 \mathrm{D}+00 \quad-0.277071 \mathrm{D}+00 \quad-0.299174 \mathrm{D}+00 \quad-0.278568 \mathrm{D}+00$

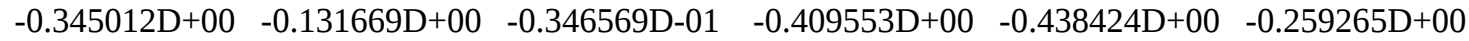

$-0.709110 \mathrm{D}+00 \quad-0.621743 \mathrm{D}+00 \quad-0.511303 \mathrm{D}+00 \quad-0.101529 \mathrm{D}+01 \quad-0.717592 \mathrm{D}+00 \quad-0.374097 \mathrm{D}+00 \quad-0.754454 \mathrm{D}+00$

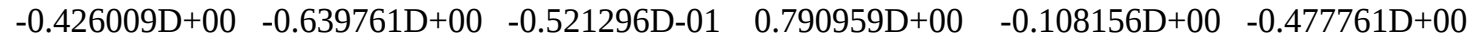

$-0.481564 \mathrm{D}+00 \quad-0.359800 \mathrm{D}+00 \quad-0.303384 \mathrm{D}+00 \quad-0.235360 \mathrm{D}+00 \quad 0.152857 \mathrm{D}+00 \quad-0.181444 \mathrm{D}+00 \quad-0.899885 \mathrm{D}+00$ $\begin{array}{llllll}-0.127039 D+01 & 0.118033 \mathrm{D}+00 & -0.535944 \mathrm{D}+00 & -0.347109 \mathrm{D}+00 & 0.318221 \mathrm{D}+00 & -0.181012 \mathrm{D}+01\end{array}$ $0.107606 \mathrm{D}+00 \quad-0.216580 \mathrm{D}+00 \quad-0.190069 \mathrm{D}+00 \quad-0.162120 \mathrm{D}+01 \quad-0.881195 \mathrm{D}+00 \quad-0.242456 \mathrm{D}-01$

$-0.162993 \mathrm{D}+01 \quad-0.299134 \mathrm{D}+00 \quad-0.277621 \mathrm{D}+01 \quad-0.207341 \mathrm{D}+00 \quad-0.428715 \mathrm{D}-01 \quad-0.184785 \mathrm{D}+00 \quad-0.550051 \mathrm{D}-01$ $-0.251041 \mathrm{D}+00 \quad-0.771750 \mathrm{D}+00 \quad-0.734069 \mathrm{D}-01 \quad-0.167536 \mathrm{D}+00$

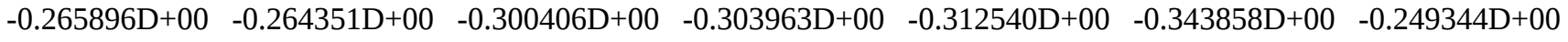
$\begin{array}{llllll}-0.316368 \mathrm{D}+00 & -0.151012 \mathrm{D}+00 & -0.605526 \mathrm{D}-01 & -0.535010 \mathrm{D}+00 & -0.657712 \mathrm{D}+00 & -0.304740 \mathrm{D}+00\end{array}$

$-0.474428 \mathrm{D}+00 \quad-0.336270 \mathrm{D}+00 \quad-0.246939 \mathrm{D}+00 \quad-0.215378 \mathrm{D}+01 \quad 0.437583 \mathrm{D}+00 \quad-0.125104 \mathrm{D}+01 \quad-0.857060 \mathrm{D}+00$ $\begin{array}{llllll}-0.322729 \mathrm{D}+00 & -0.350915 \mathrm{D}+00 & 0.416497 \mathrm{D}-01 & -0.137540 \mathrm{D}+00 & -0.335831 \mathrm{D}+00 & 0.112865 \mathrm{D}+01\end{array}$

$\begin{array}{lllllll}-0.355396 \mathrm{D}+00 & -0.411012 \mathrm{D}+00 & 0.826618 \mathrm{D}-01 & -0.179825 \mathrm{D}-01 & -0.135902 \mathrm{D}+00 & 0.200580 \mathrm{D}+00 & 0.203993 \mathrm{D}+00\end{array}$ $\begin{array}{llllll}0.926078 D-01 & -0.631747 \mathrm{D}+00 & 0.111805 \mathrm{D}+01 & -0.147789 \mathrm{D}+01 & -0.895819 \mathrm{D}+00 & -0.102173 \mathrm{D}+01\end{array}$

$-0.239895 \mathrm{D}+00-0.308355 \mathrm{D}+00 \quad-0.522630 \mathrm{D}+00 \quad-0.122772 \mathrm{D}+01 \quad-0.879214 \mathrm{D}+00 \quad-0.779021 \mathrm{D}+00 \quad-0.120249 \mathrm{D}+01$ $\begin{array}{llllll}-0.107269 \mathrm{D}+01 & -0.346374 \mathrm{D}+01 & -0.184173 \mathrm{D}+00 & -0.888925 \mathrm{D}+00 & 0.526583 \mathrm{D}-01 & 0.105479 \mathrm{D}+00\end{array}$ $\begin{array}{lllll}0.245052 \mathrm{D}+00 & -0.950747 \mathrm{D}+00 & -0.122034 \mathrm{D}+00 & -0.521815 \mathrm{D}+00 & -0.135364 \mathrm{D}+00\end{array}$ 
$-0.116673 \mathrm{D}+00 \quad-0.112137 \mathrm{D}+00 \quad-0.150659 \mathrm{D}+00 \quad-0.151223 \mathrm{D}+00 \quad-0.228567 \mathrm{D}+00 \quad-0.193532 \mathrm{D}+00 \quad-0.256846 \mathrm{D}+00$

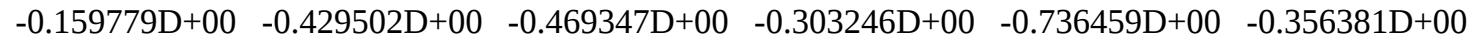

$-0.483328 \mathrm{D}+00 \quad-0.363242 \mathrm{D}+00 \quad-0.173240 \mathrm{D}+00 \quad-0.215037 \mathrm{D}+00 \quad-0.491489 \mathrm{D}-01 \quad-0.323836 \mathrm{D}+00 \quad-0.821811 \mathrm{D}+00$ $\begin{array}{llllll}0.168365 \mathrm{D}-01 & -0.948873 \mathrm{D}+00 & 0.653923 \mathrm{D}+00 & -0.203445 \mathrm{D}+00 & 0.161147 \mathrm{D}+01 & -0.310049 \mathrm{D}+00\end{array}$

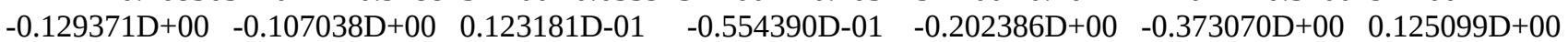
0.846581D-01 $\quad-0.487092 \mathrm{D}+00 \quad-0.376912 \mathrm{D}+00 \quad-0.720957 \mathrm{D}+00 \quad-0.506300 \mathrm{D}+00 \quad-0.217744 \mathrm{D}+01$

$-0.307423 \mathrm{D}+00 \quad-0.180774 \mathrm{D}+00 \quad-0.231643 \mathrm{D}+01 \quad-0.912016 \mathrm{D}+00 \quad-0.115118 \mathrm{D}+01 \quad-0.502088 \mathrm{D}+00 \quad-0.321563 \mathrm{D}+01$ $\begin{array}{llllll}-0.421636 \mathrm{D}+00 & -0.312600 \mathrm{D}+01 & -0.696415 \mathrm{D}+00 & -0.622831 \mathrm{D}+00 & -0.443791 \mathrm{D}+00 & 0.236888 \mathrm{D}+00\end{array}$ $\begin{array}{llllll}0.139507 \mathrm{D}+00 & -0.191016 \mathrm{D}+00 & -0.221847 \mathrm{D}+00 & -0.408310 \mathrm{D}+00 & -0.686865 \mathrm{D}-01 & -0.217210 \mathrm{D}-01\end{array}$

-0.394779D-01 -0.431794D-01 -0.819991D-02 $\quad$-0.118273D-01 0.572851D-01 $\quad 0.513867 \mathrm{D}-01 \quad 0.361029 \mathrm{D}-01$ $\begin{array}{llllll}0.805804 \mathrm{D}-02 & 0.119222 \mathrm{D}+00 & 0.149687 \mathrm{D}+00 & -0.490993 \mathrm{D}+00 & -0.595749 \mathrm{D}+00 & -0.991706 \mathrm{D}+00\end{array}$

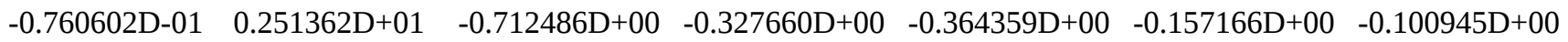
$\begin{array}{llllll}-0.181678 \mathrm{D}+00 & -0.955189 \mathrm{D}+00 & 0.251376 \mathrm{D}+00 & -0.289498 \mathrm{D}-02 & 0.304175 \mathrm{D}+00 & -0.202403 \mathrm{D}-01\end{array}$

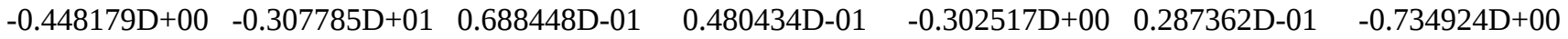
0.612829D-01 $\quad-0.626696 \mathrm{D}-01 \quad 0.131799 \mathrm{D}-01 \quad-0.639434 \mathrm{D}-01 \quad-0.590692 \mathrm{D}-01 \quad-0.103136 \mathrm{D}+00$

$-0.270059 \mathrm{D}+00 \quad-0.574066 \mathrm{D}+00 \quad-0.154790 \mathrm{D}+00 \quad-0.878099 \mathrm{D}-01 \quad-0.943632 \mathrm{D}-01 \quad 0.624638 \mathrm{D}-01 \quad-0.794334 \mathrm{D}-02$ $\begin{array}{llllll}-0.818569 \mathrm{D}-01 & 0.729773 \mathrm{D}-02 & -0.365161 \mathrm{D}+00 & -0.206466 \mathrm{D}+00 & -0.159440 \mathrm{D}+00 & 0.630661 \mathrm{D}+00\end{array}$

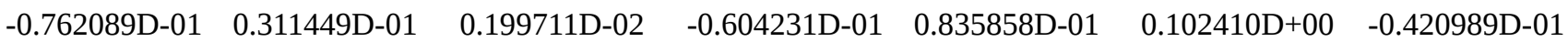

$\begin{array}{lllllll}-0.542118 D-01 & -0.585487 D-01 & -0.168425 D-01 & -0.220112 D-01 & 0.654466 D-01 & 0.534849 D-01 & 0.230966 D-01\end{array}$ 0.638621D-02 0.295494D-01 0.555114D-01 $\quad-0.452025 \mathrm{D}+00 \quad-0.896342 \mathrm{D}+00 \quad-0.826158 \mathrm{D}+00$

$-0.558154 \mathrm{D}+00 \quad-0.182502 \mathrm{D}+00 \quad-0.193529 \mathrm{D}+00 \quad-0.170835 \mathrm{D}+00 \quad 0.201364 \mathrm{D}-01 \quad-0.752025 \mathrm{D}+00 \quad-0.346557 \mathrm{D}+00$ $\begin{array}{llllll}-0.115835 \mathrm{D}+00 & -0.598916 \mathrm{D}+00 & -0.572268 \mathrm{D}+00 & -0.256403 \mathrm{D}+00 & 0.688065 \mathrm{D}+00 & 0.235716 \mathrm{D}+00\end{array}$ $\begin{array}{llllll}0.575472 \mathrm{D}+00 & 0.512693 \mathrm{D}+00 & 0.234593 \mathrm{D}+00 & -0.360146 \mathrm{D}-01 & 0.125057 \mathrm{D}+00 & 0.295833 \mathrm{D}+00\end{array}$ $\begin{array}{llllll}0.340809 \mathrm{D}+00 & -0.947947 \mathrm{D}-01 & 0.561164 \mathrm{D}-01 & 0.487963 \mathrm{D}-01 & -0.861960 \mathrm{D}-02 & -0.160042 \mathrm{D}-01\end{array}$

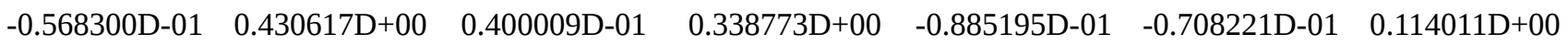
0.773342D-01 $\quad 0.637784 \mathrm{D}-02 \quad-0.100268 \mathrm{D}+00 \quad-0.390363 \mathrm{D}+00 \quad 0.744531 \mathrm{D}-01 \quad 0.793405 \mathrm{D}-01$

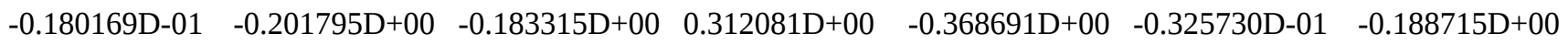
$-0.119398 \mathrm{D}+00 \quad 0.126198 \mathrm{D}+00$

$-0.118246 \mathrm{D}+00 \quad-0.121096 \mathrm{D}+00 \quad-0.988533 \mathrm{D}-01 \quad-0.101614 \mathrm{D}+00 \quad-0.563140 \mathrm{D}-01 \quad-0.613334 \mathrm{D}-01 \quad-0.579929 \mathrm{D}-01$

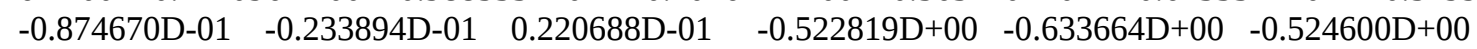

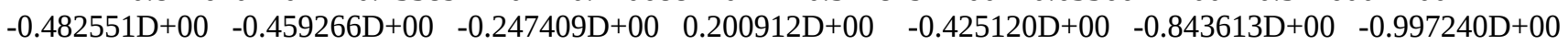
$0.116718 \mathrm{D}+01 \quad-0.865080 \mathrm{D}-01 \quad 0.146849 \mathrm{D}+01 \quad-0.108878 \mathrm{D}+00 \quad-0.428422 \mathrm{D}+00 \quad-0.365086 \mathrm{D}+00$

$-0.365784 \mathrm{D}+00 \quad 0.276832 \mathrm{D}+01 \quad 0.442298 \mathrm{D}-02 \quad-0.121425 \mathrm{D}+00 \quad-0.123181 \mathrm{D}+01 \quad-0.495735 \mathrm{D}+00 \quad-0.113276 \mathrm{D}+00$ $\begin{array}{llllll}-0.572296 \mathrm{D}-01 & -0.687085 \mathrm{D}+00 & -0.182344 \mathrm{D}+01 & -0.572164 \mathrm{D}+00 & -0.100733 \mathrm{D}+01 & -0.279183 \mathrm{D}+01\end{array}$ $0.184735 \mathrm{D}+00 \quad-0.651546 \mathrm{D}-01 \quad-0.102739 \mathrm{D}+01 \quad-0.759487 \mathrm{D}+00 \quad-0.104369 \mathrm{D}+01-0.331190 \mathrm{D}+00$

$-0.734904 \mathrm{D}+00-0.277371 \mathrm{D}+01 \quad-0.597785 \mathrm{D}+00 \quad-0.992722 \mathrm{D}+00 \quad-0.914360 \mathrm{D}+00 \quad-0.111479 \mathrm{D}+00 \quad 0.125340 \mathrm{D}+00$

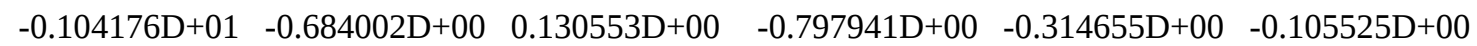

$-0.944753 \mathrm{D}-01 \quad-0.327867 \mathrm{D}+00 \quad 0.129481 \mathrm{D}+00$

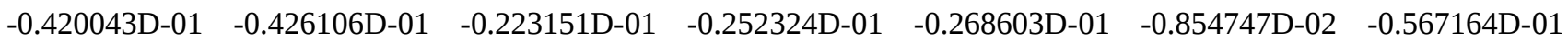

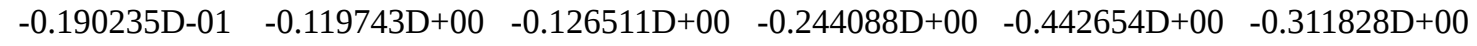

$-0.305671 \mathrm{D}+00 \quad-0.412675 \mathrm{D}+00 \quad-0.102382 \mathrm{D}+00 \quad-0.551982 \mathrm{D}-01 \quad-0.190241 \mathrm{D}+00 \quad-0.290393 \mathrm{D}+00 \quad-0.266020 \mathrm{D}+00$

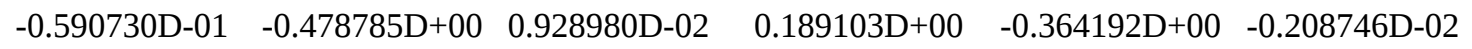

$-0.242889 \mathrm{D}+00 \quad 0.532920 \mathrm{D}-01 \quad-0.148135 \mathrm{D}+00 \quad-0.154912 \mathrm{D}+00 \quad-0.147087 \mathrm{D}+00 \quad-0.100995 \mathrm{D}-01 \quad-0.314646 \mathrm{D}+00$ $-0.139405 \mathrm{D}+01 \quad-0.477468 \mathrm{D}+00 \quad-0.357182 \mathrm{D}+00 \quad-0.546605 \mathrm{D}+00 \quad-0.485824 \mathrm{D}+00 \quad-0.846296 \mathrm{D}+00$

$-0.160238 \mathrm{D}+00 \quad 0.164778 \mathrm{D}+00 \quad-0.732889 \mathrm{D}+00 \quad-0.138208 \mathrm{D}+01 \quad-0.801459 \mathrm{D}+00 \quad-0.123714 \mathrm{D}-01 \quad-0.361205 \mathrm{D}+00$

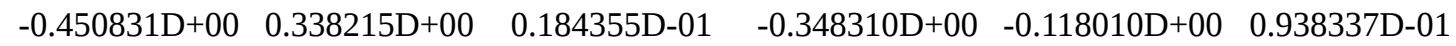

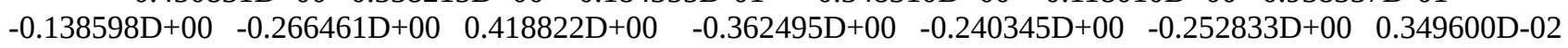
$-0.328923 \mathrm{D}+00 \quad-0.453422 \mathrm{D}+00 \quad-0.588034 \mathrm{D}-01$

$\begin{array}{lllllll}-0.877202 D-01 & -0.905816 \mathrm{D}-01 & -0.851929 \mathrm{D}-01 & -0.853000 \mathrm{D}-01 & -0.676891 \mathrm{D}-01 & -0.707481 \mathrm{D}-01 & -0.712293 \mathrm{D}-01\end{array}$

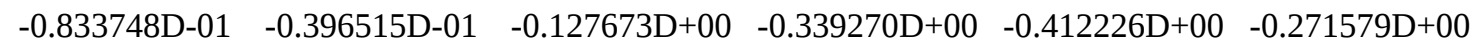

$-0.162846 \mathrm{D}+00 \quad-0.340973 \mathrm{D}+00 \quad-0.224422 \mathrm{D}+00 \quad-0.992985 \mathrm{D}-01 \quad-0.174690 \mathrm{D}+00 \quad-0.262546 \mathrm{D}+00 \quad-0.960335 \mathrm{D}-01$ 0.730685D-01 -0.112055D+00 -0.747140D-01 $-0.982124 \mathrm{D}-01 \quad-0.387024 \mathrm{D}-01 \quad 0.397290 \mathrm{D}-01$

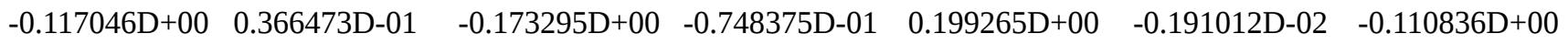
$\begin{array}{llllll}-0.140303 \mathrm{D}+00 & -0.146220 \mathrm{D}+00 & -0.202415 \mathrm{D}+00 & -0.133075 \mathrm{D}+00 & -0.490960 \mathrm{D}-01 & -0.800854 \mathrm{D}+00\end{array}$ $\begin{array}{llllll}0.247577 \mathrm{D}+00 & 0.878613 \mathrm{D}-01 & -0.863682 \mathrm{D}+00 & -0.966423 \mathrm{D}+00 & -0.641093 \mathrm{D}+00 & 0.322686 \mathrm{D}+00\end{array}$ 


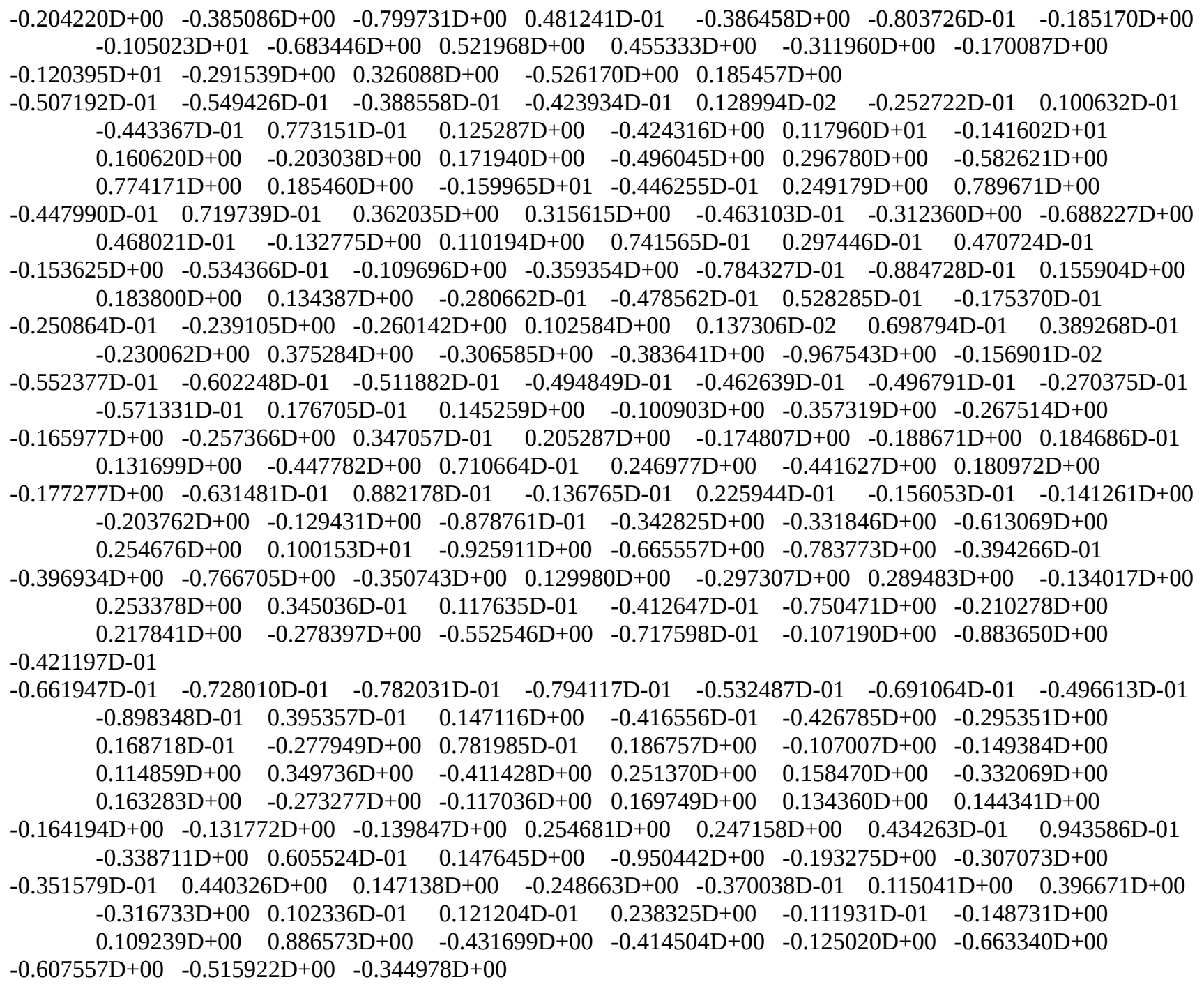


C10H8

MP2/cc-pvdz level

anharmonic

48, 0, 0, "We"

167.9063

182.1227

355.792

377.3892

451.0864

463.1679

505.6892

514.2777

534.7599

558.5015

618.0468

721.4317

771.0791

784.7772

802.6538

837.6334

849.8846

924.4933

930.9759

932.0827

933.3046

950.9371

1042.2167

1050.9334

1138.5517

1161.0417

1169.8178

1170.2174

1254.6356

1254.8459

1276.8814

1406.4214

1458.2545

1484.0106

1489.9606

1494.6364

1561.415

1624.7355

1637.0164

1688.3771

3202.1105

3203.5013

3206.3817

3208.4972

3224.0913

3224.595

3237.636

3238.4594

lower

$-11.405$ 


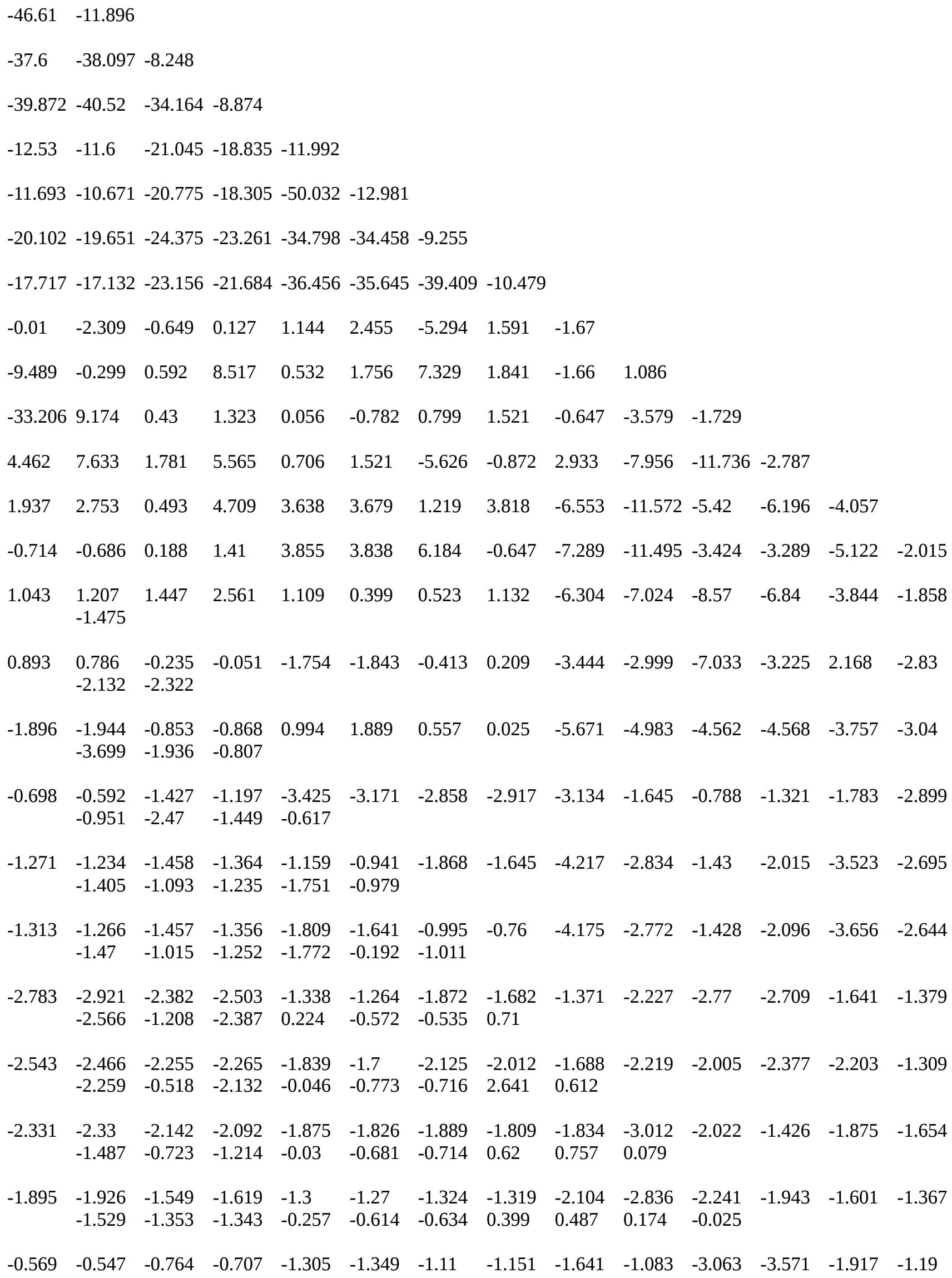




\begin{tabular}{|c|c|c|c|c|c|c|c|c|c|c|c|c|c|}
\hline & -0.963 & -1.851 & -0.741 & -0.549 & -0.518 & -0.518 & 0.02 & -0.14 & -0.446 & -0.213 & -0.306 & & \\
\hline \multirow[t]{2}{*}{-0.588} & -0.573 & -0.67 & -0.641 & -0.925 & -0.901 & -0.805 & -0.82 & -1.496 & -1.575 & -3.837 & -2.961 & -1.876 & -1.178 \\
\hline & -0.614 & -1.733 & -0.818 & -0.415 & -0.47 & -0.474 & -0.078 & -0.144 & -0.681 & -0.403 & -1.831 & -0.442 & \\
\hline \multirow[t]{2}{*}{-3.571} & -3.625 & -2.985 & -3.097 & -1.56 & -1.492 & -1.921 & -1.802 & -0.526 & -1.132 & -1.136 & -0.671 & -0.285 & 1.279 \\
\hline & -0.315 & -0.367 & -0.891 & 0.103 & 0.305 & 0.276 & -0.561 & -0.639 & -0.223 & 0.265 & -0.619 & -0.708 & 1.877 \\
\hline \multirow[t]{3}{*}{-3.579} & -3.628 & -3.012 & -3.12 & -1.664 & -1.601 & -2.002 & -1.886 & -1.166 & -1.186 & -1.143 & -0.657 & -0.08 & -0.319 \\
\hline & -0.083 & -0.214 & 0.874 & 0.292 & 0.495 & 0.41 & -0.482 & -0.742 & 0.108 & 0.228 & -0.65 & -0.671 & 10.433 \\
\hline & 4.191 & & & & & & & & & & & & \\
\hline \multirow[t]{3}{*}{-2.071} & -2.047 & -2.246 & -2.187 & -2.697 & -2.699 & -2.451 & -2.467 & -1.444 & -1.518 & -1.066 & -0.643 & 0.015 & -0.454 \\
\hline & -1.901 & -0.194 & 0.688 & 0.081 & 0.31 & 0.251 & 0.162 & -0.319 & -0.523 & -0.332 & -0.113 & -0.175 & 2.313 \\
\hline & 4.314 & 1.871 & & & & & & & & & & & \\
\hline \multirow[t]{3}{*}{-0.66} & -0.649 & -0.657 & -0.625 & -0.844 & -0.821 & -0.732 & -0.701 & -5.101 & -1.43 & -1.999 & -1.304 & -1.615 & -0.958 \\
\hline & -0.217 & -1.385 & -0.435 & -0.218 & 0.125 & 0.057 & -0.203 & -0.231 & -0.271 & -0.156 & -0.262 & -0.314 & 0.549 \\
\hline & 1.106 & 0.512 & 0.367 & & & & & & & & & & \\
\hline \multirow[t]{3}{*}{-2.389} & -2.382 & -2.406 & -2.396 & -2.429 & -2.432 & -2.284 & -2.292 & -1.158 & -1.604 & -1.334 & -0.534 & 0.308 & -0.545 \\
\hline & -0.03 & -0.354 & 0.161 & 0.182 & 0.199 & 0.169 & 0.015 & -0.148 & -0.563 & -0.212 & -0.162 & -0.188 & 1.744 \\
\hline & 4.286 & 4.449 & 0.386 & 1.311 & & & & & & & & & \\
\hline \multirow[t]{3}{*}{-1.41} & -1.362 & -1.766 & -1.66 & -2.919 & -2.947 & -2.494 & -2.599 & -1.135 & -0.541 & -1.225 & -0.948 & 0.074 & -0.109 \\
\hline & -0.478 & -0.112 & 0.13 & 0.094 & 0.531 & 0.346 & 0.386 & 0.22 & 0.311 & 0.256 & -0.156 & 0.956 & 0.664 \\
\hline & 1.701 & 2.013 & -0.016 & -0.136 & 0.652 & & & & & & & & \\
\hline \multirow[t]{3}{*}{-1.588} & -1.55 & -1.812 & -1.737 & -2.634 & -2.677 & -2.314 & -2.411 & -0.988 & -0.97 & -1.221 & -0.874 & 0.513 & -0.363 \\
\hline & -0.381 & -0.236 & 0.116 & 0.245 & 0.197 & 0.1 & 0.479 & 0.249 & 0.381 & 0.186 & 0.217 & -0.331 & 0.063 \\
\hline & 1.439 & -0.537 & -0.268 & 0.459 & -0.214 & 0.094 & & & & & & & \\
\hline \multirow[t]{3}{*}{-0.739} & -0.761 & -0.567 & -0.608 & -0.175 & -0.141 & -0.259 & -0.213 & -2.159 & -0.981 & -0.231 & -1.448 & -1.752 & -1.39 \\
\hline & -0.763 & -0.614 & -0.343 & -0.629 & -0.359 & -0.408 & 0.672 & 0.022 & 0.142 & 0.144 & -0.686 & -0.657 & 0.205 \\
\hline & 0.658 & 0.147 & 0.333 & 0.083 & -0.11 & -0.132 & -0.147 & & & & & & \\
\hline \multirow[t]{3}{*}{-2.179} & -2.193 & -2 & -2.033 & -1.541 & -1.52 & -1.655 & -1.596 & -1.224 & -0.347 & -0.777 & -0.592 & -0.078 & -0.237 \\
\hline & -0.347 & -0.154 & 0.188 & 0.065 & 0.252 & 0.337 & 0.876 & 0.777 & 0.642 & -0.079 & 0.001 & -0.048 & -0.341 \\
\hline & 0.282 & 1.762 & -0.921 & 0.067 & 0.613 & -0.336 & -0.281 & 0.228 & & & & & \\
\hline \multirow[t]{3}{*}{-0.245} & -0.244 & -0.312 & -0.305 & -0.386 & -0.39 & -0.366 & -0.374 & -4.568 & -0.755 & 0.504 & -0.758 & -0.117 & -1.121 \\
\hline & -0.374 & -5.49 & -0.558 & -0.719 & -0.494 & -0.435 & -0.021 & -0.006 & -0.186 & 0.303 & -0.366 & -0.357 & -0.18 \\
\hline & -0.065 & -0.225 & 3.361 & -0.156 & -0.161 & -0.069 & -0.029 & -0.246 & -0.42 & & & & \\
\hline \multirow{3}{*}{-1.925} & -1.924 & -1.84 & -1.848 & -1.65 & -1.643 & -1.677 & -1.654 & -0.29 & -0.718 & -0.91 & -0.256 & -0.125 & -0.45 \\
\hline & -0.19 & -0.442 & 0.087 & 0.231 & 0.364 & 0.386 & 1.465 & 0.246 & -1.618 & 0.351 & -0.028 & 0.133 & -1.439 \\
\hline & 0.442 & -0.236 & -0.709 & 0.056 & -0.785 & -0.549 & -0.248 & -0.45 & -0.102 & -0.137 & & & \\
\hline \multirow[t]{3}{*}{-0.168} & -0.166 & -0.24 & -0.24 & -0.215 & -0.2 & -0.224 & -0.214 & -1.362 & -1.02 & -1.502 & -1.241 & -0.958 & -1.091 \\
\hline & -0.697 & -0.984 & -0.498 & -0.48 & -0.317 & -0.326 & 0.104 & 0.062 & 0.131 & -0.123 & 0.188 & 0.35 & -0.153 \\
\hline & -0.03 & 0.079 & -0.217 & 0.025 & -0.346 & -0.386 & 0.004 & -0.051 & -0.232 & 0.011 & 0.155 & & \\
\hline \multirow[t]{3}{*}{-0.376} & -0.377 & -0.333 & -0.324 & -0.268 & -0.244 & -0.176 & -0.163 & -0.851 & -0.885 & -1.472 & -0.535 & -1.164 & -0.346 \\
\hline & -0.836 & -0.61 & 0.75 & 0.62 & 0.984 & 0.942 & 0.091 & 0.165 & 0.405 & 0.577 & -2.499 & 0.421 & 12.636 \\
\hline & 21.37 & 8.339 & 1.473 & 6.843 & 4.423 & 3.441 & 1.103 & 3.175 & 0.163 & 2.687 & 0.242 & 10.608 & \\
\hline \multirow[t]{2}{*}{-0.004} & 0.011 & 0.084 & 0.135 & 0.218 & 0.422 & 0.505 & 0.753 & -0.51 & -0 . & -1.067 & -0.005 & 0.322 & 1.330 \\
\hline & 1.98 & -0.176 & 2.958 & 4.44 & 4.852 & 4.344 & 0.468 & 0.591 & 1.206 & 4.102 & -3.565 & -1.927 & 29.281 \\
\hline
\end{tabular}




\begin{tabular}{|c|c|c|c|c|c|c|c|c|c|c|c|c|c|}
\hline & 52.568 & 36.112 & 12.931 & 24.831 & 36.988 & 16.627 & 3.888 & 20.442 & -3.05 & 10.671 & 2.348 & 103.66 & 203.70 \\
\hline-0.086 & $\begin{array}{l}-0.095 \\
-0.312 \\
-0.084 \\
-0.058\end{array}$ & $\begin{array}{l}-0.074 \\
-0.174 \\
-0.067\end{array}$ & $\begin{array}{l}-0.094 \\
-0.135 \\
-0.444\end{array}$ & $\begin{array}{l}0.057 \\
-0.436 \\
-0.079\end{array}$ & $\begin{array}{l}0.039 \\
-0.138 \\
-0.236\end{array}$ & $\begin{array}{l}0.009 \\
-0.171 \\
-0.191\end{array}$ & $\begin{array}{l}0.003 \\
0.109 \\
-0.319\end{array}$ & $\begin{array}{l}-1.547 \\
0.13 \\
-0.073\end{array}$ & $\begin{array}{l}-1.624 \\
-0.377 \\
-0.536\end{array}$ & $\begin{array}{l}-0.143 \\
0.673 \\
-0.176\end{array}$ & $\begin{array}{l}-0.953 \\
-0.05 \\
0.181\end{array}$ & $\begin{array}{l}-1.446 \\
-0.119 \\
-0.059\end{array}$ & $\begin{array}{l}-0.773 \\
-0.199 \\
1.18\end{array}$ \\
\hline-0.14 & $\begin{array}{l}-0.138 \\
-0.363 \\
0.08 \\
-0.037\end{array}$ & $\begin{array}{l}-0.142 \\
-1.673 \\
-0.063 \\
0.053\end{array}$ & $\begin{array}{l}-0.131 \\
-0.371 \\
0.07\end{array}$ & $\begin{array}{l}-0.244 \\
-0.125 \\
-0.116\end{array}$ & $\begin{array}{l}-0.234 \\
-0.492 \\
0.156\end{array}$ & $\begin{array}{l}-0.224 \\
-0.484 \\
0.025\end{array}$ & $\begin{array}{l}-0.216 \\
-0.169 \\
0.395\end{array}$ & $\begin{array}{l}-0.197 \\
0.114 \\
-0.037\end{array}$ & $\begin{array}{l}-0.628 \\
0.144 \\
-0.094\end{array}$ & $\begin{array}{l}-1.238 \\
0.245 \\
0.131\end{array}$ & $\begin{array}{l}-0.708 \\
-0.221 \\
0.018\end{array}$ & $\begin{array}{l}-0.721 \\
-0.439 \\
0.421\end{array}$ & $\begin{array}{l}-0.712 \\
0.007 \\
3.599\end{array}$ \\
\hline-0.184 & $\begin{array}{l}-0.179 \\
-0.145 \\
1.488 \\
0.222\end{array}$ & $\begin{array}{l}-0.213 \\
-0.894 \\
2.918 \\
-0.844\end{array}$ & $\begin{array}{l}-0.202 \\
-1.059 \\
-0.725 \\
1.65\end{array}$ & $\begin{array}{l}-0.295 \\
-0.024 \\
0.841\end{array}$ & $\begin{array}{l}-0.307 \\
-0.312 \\
1.138\end{array}$ & $\begin{array}{l}-0.259 \\
-0.321 \\
-0.003\end{array}$ & $\begin{array}{l}-0.269 \\
-0.207 \\
-0.323\end{array}$ & $\begin{array}{l}-0.995 \\
-0.937 \\
1.57\end{array}$ & $\begin{array}{l}-0.412 \\
-0.118 \\
-1.709\end{array}$ & $\begin{array}{l}-0.746 \\
0.884 \\
0.79\end{array}$ & $\begin{array}{l}-0.611 \\
0.198 \\
0.469\end{array}$ & $\begin{array}{l}-0.371 \\
0.211 \\
4.479\end{array}$ & $\begin{array}{l}-0.381 \\
1.943 \\
29.514\end{array}$ \\
\hline-0.251 & $\begin{array}{l}-0.247 \\
-0.43 \\
2.277 \\
-0.197\end{array}$ & $\begin{array}{l}-0.194 \\
-0.912 \\
1.219 \\
0.833\end{array}$ & $\begin{array}{l}-0.192 \\
0.764 \\
0.57 \\
2.519\end{array}$ & $\begin{array}{l}-0.136 \\
0.221 \\
1.283 \\
4.014\end{array}$ & $\begin{array}{l}-0.141 \\
0.016 \\
3.306\end{array}$ & $\begin{array}{l}-0.124 \\
-0.008 \\
-0.381\end{array}$ & $\begin{array}{l}-0.117 \\
-0.541 \\
0.558\end{array}$ & $\begin{array}{l}-0.603 \\
-0.381 \\
1.455\end{array}$ & $\begin{array}{l}-0.34 \\
-2.535 \\
-2.048\end{array}$ & $\begin{array}{l}-1.17 \\
-0.487 \\
2.533\end{array}$ & $\begin{array}{l}-0.657 \\
0.813 \\
-0.233\end{array}$ & $\begin{array}{l}-0.19 \\
3.047 \\
2.861\end{array}$ & $\begin{array}{l}-0.136 \\
1.233 \\
80.568\end{array}$ \\
\hline-0.108 & $\begin{array}{l}-0.108 \\
0.061 \\
0.861 \\
-0.226\end{array}$ & $\begin{array}{l}-0.072 \\
-1.151 \\
0.794 \\
-0.182\end{array}$ & $\begin{array}{l}-0.082 \\
0.034 \\
1.262 \\
1.143\end{array}$ & $\begin{array}{l}0.024 \\
-0.56 \\
1.037 \\
1.21\end{array}$ & $\begin{array}{l}0.032 \\
0.818 \\
-0.986 \\
-0.256\end{array}$ & $\begin{array}{l}0.01 \\
0.837 \\
-0.137\end{array}$ & $\begin{array}{l}0.014 \\
-0.102 \\
-0.324\end{array}$ & $\begin{array}{l}-0.924 \\
-0.139 \\
-1.528\end{array}$ & $\begin{array}{l}-0.854 \\
-0.337 \\
2.602\end{array}$ & $\begin{array}{l}-0.72 \\
-0.193 \\
-0.18\end{array}$ & $\begin{array}{l}-0.426 \\
-0.331 \\
0.387\end{array}$ & $\begin{array}{l}-0.552 \\
0.074 \\
3.507\end{array}$ & $\begin{array}{l}-0.92 \\
0.479 \\
10.595\end{array}$ \\
\hline-0.002 & $\begin{array}{l}-0.018 \\
-0.52 \\
-0.129 \\
0.279\end{array}$ & $\begin{array}{l}0.054 \\
-0.458 \\
0.049 \\
-0.005\end{array}$ & $\begin{array}{l}0.005 \\
-0.303 \\
0.184 \\
0.177\end{array}$ & $\begin{array}{l}0.172 \\
0.73 \\
-0.065 \\
0.049\end{array}$ & $\begin{array}{l}0.039 \\
-0.184 \\
0.035 \\
-0.221\end{array}$ & $\begin{array}{l}0.131 \\
-0.208 \\
-0.014 \\
0.218\end{array}$ & $\begin{array}{l}0.031 \\
0.99 \\
-0.97\end{array}$ & $\begin{array}{l}-1.41 \\
0.082 \\
-0.206\end{array}$ & $\begin{array}{l}-0.712 \\
0.296 \\
-0.576\end{array}$ & $\begin{array}{l}-0.328 \\
0.769 \\
0.133\end{array}$ & $\begin{array}{l}-0.47 \\
0.779 \\
-0.041\end{array}$ & $\begin{array}{l}-0.607 \\
-0.082 \\
0.042\end{array}$ & $\begin{array}{l}-0.256 \\
-0.08 \\
1.568\end{array}$ \\
\hline-0.024 & $\begin{array}{l}-0.018 \\
-0.136 \\
-0.11 \\
-0.261\end{array}$ & $\begin{array}{l}-0.011 \\
-0.092 \\
-0.247 \\
0.144\end{array}$ & $\begin{array}{l}0 \\
-0.23 \\
-0.326 \\
0.067\end{array}$ & $\begin{array}{l}-0.017 \\
-0.323 \\
-0.316 \\
0.553\end{array}$ & $\begin{array}{l}0.02 \\
-0.007 \\
-1.688 \\
0.205\end{array}$ & $\begin{array}{l}-0.001 \\
0.043 \\
-0.687 \\
-0.51\end{array}$ & $\begin{array}{l}0.027 \\
-0.199 \\
0.012 \\
0.048\end{array}$ & $\begin{array}{l}-0.321 \\
-0.121 \\
0.19\end{array}$ & $\begin{array}{l}-0.403 \\
-0.088 \\
0.201\end{array}$ & $\begin{array}{l}-0.429 \\
-0.116 \\
-0.219\end{array}$ & $\begin{array}{l}-0.26 \\
0.064 \\
-0.214\end{array}$ & $\begin{array}{l}-0.445 \\
1.072 \\
0.706\end{array}$ & $\begin{array}{l}-0.362 \\
-0.448 \\
3.465\end{array}$ \\
\hline-0.04 & $\begin{array}{l}-0.041 \\
-0.238 \\
0.01 \\
0.839\end{array}$ & $\begin{array}{l}-0.041 \\
-0.067 \\
-0.068 \\
0.264\end{array}$ & $\begin{array}{l}-0.039 \\
-0.133 \\
0.093 \\
0.084\end{array}$ & $\begin{array}{l}-0.051 \\
-0.035 \\
0.033 \\
0.289\end{array}$ & $\begin{array}{l}-0.06 \\
-0.172 \\
-0.118 \\
-0.22\end{array}$ & $\begin{array}{l}-0.04 \\
-0.095 \\
-0.18 \\
0.432\end{array}$ & $\begin{array}{l}-0.044 \\
0.071 \\
0.619 \\
-0.2\end{array}$ & $\begin{array}{l}-0.43 \\
0.073 \\
-0.202 \\
-0.17\end{array}$ & $\begin{array}{l}-0.293 \\
0.131 \\
0.243\end{array}$ & $\begin{array}{l}-0.205 \\
0.108 \\
-0.187\end{array}$ & $\begin{array}{l}-0.308 \\
-0.005 \\
0.029\end{array}$ & $\begin{array}{l}-0.334 \\
-0.019 \\
0.699\end{array}$ & $\begin{array}{l}-0.167 \\
-0.425 \\
3.043\end{array}$ \\
\hline
\end{tabular}




\section{$\mathrm{P}(\mathrm{CH} 3 \mathrm{CH} 2) 3$}

B3LYP-cc-pVdz

$5700 \mathrm{WE}$

152.5325

156.1717

170.4489

235.7745

239.1117

241.4003

322.0411

356.4796

357.4726

604.801

651.7312

652.9108

748.2174

748.8755

762.7919

975.9022

976.7756

978.0298

994.0442

994.9626

1001.3588

1057.9892

1061.2596

1061.8225

1247.7959

1249.9938

1256.298

1260.2519

1261.7234

1274.3997

1395.0645

1395.5054

1396.5304

1434.3646

1441.8555

1442.5205

1468.0774

1469.7526

1470.1028

1474.6733

1474.9711

1475.4679

3009.836

3010.5025

3011.3528

3025.4939

3025.7586

3026.6126

3061.3226

3061.7035

3063.0466

3091.5963

3092.92 


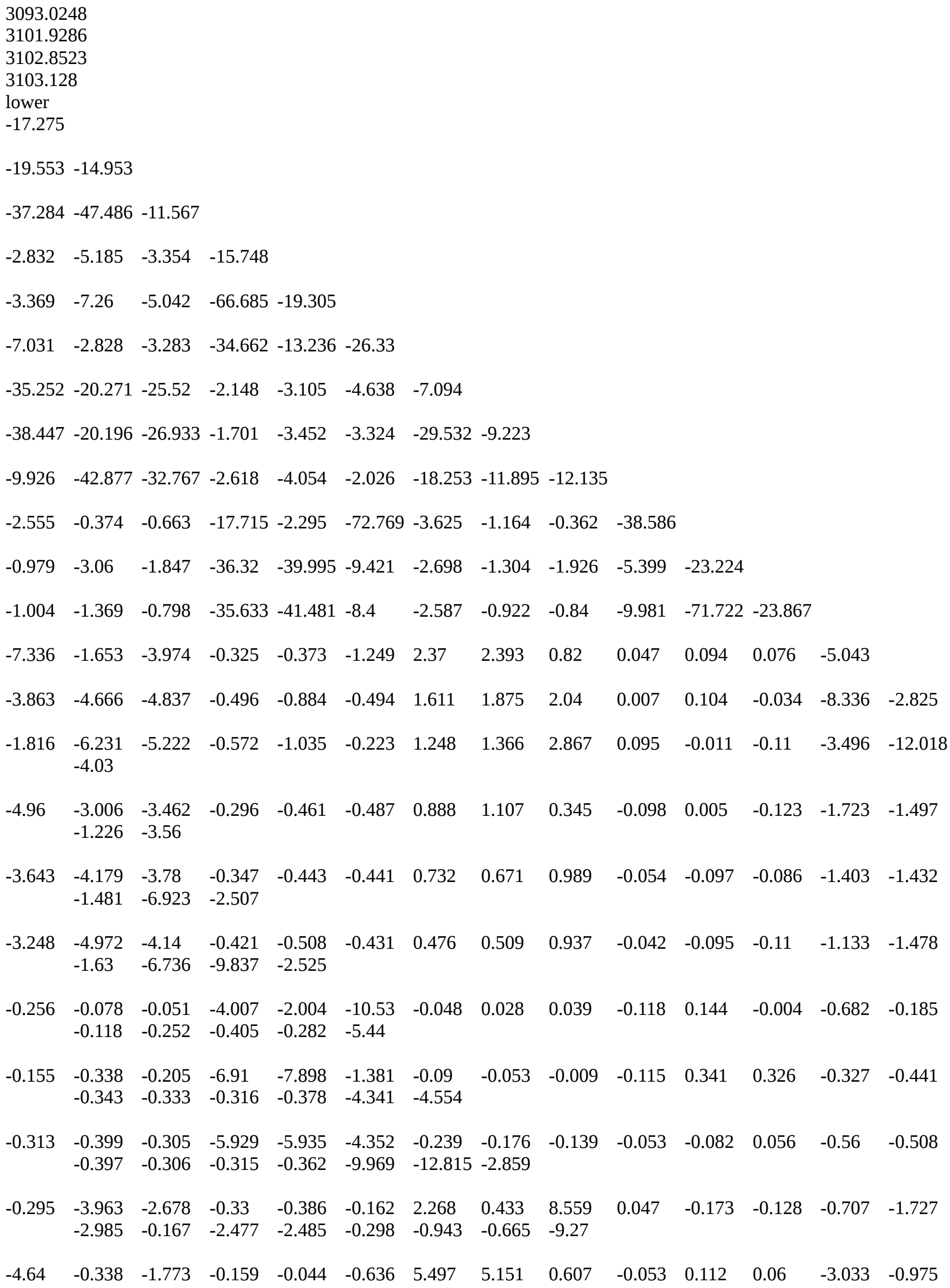




\begin{tabular}{|c|c|c|c|c|c|c|c|c|c|c|c|c|c|}
\hline & -0.093 & -1.746 & -1.657 & -1.146 & -1.19 & -0.041 & -0.404 & -0.264 & -8.663 & & & & \\
\hline-1.841 & $\begin{array}{l}-2.555 \\
-1.573\end{array}$ & $\begin{array}{l}-2.284 \\
-2.682\end{array}$ & $\begin{array}{l}-0.217 \\
-0.817\end{array}$ & $\begin{array}{l}-0.47 \\
-0.961\end{array}$ & $\begin{array}{l}-0.049 \\
-0.245\end{array}$ & $\begin{array}{l}3.259 \\
-0.951\end{array}$ & $\begin{array}{l}5.722 \\
-0.589\end{array}$ & $\begin{array}{l}2.235 \\
-0.323\end{array}$ & $\begin{array}{l}-0.029 \\
-0.972\end{array}$ & $\begin{array}{l}0.066 \\
-8.46\end{array}$ & -0.206 & -0.444 & -1.755 \\
\hline-0.429 & $\begin{array}{l}-0.408 \\
-0.845\end{array}$ & $\begin{array}{l}-0.378 \\
-0.693\end{array}$ & $\begin{array}{l}-0.745 \\
-0.706\end{array}$ & $\begin{array}{l}-0.627 \\
-0.428\end{array}$ & $\begin{array}{l}-1.197 \\
-2.031\end{array}$ & $\begin{array}{l}-0.313 \\
-0.512\end{array}$ & $\begin{array}{l}-0.308 \\
-1.022\end{array}$ & $\begin{array}{l}-0.286 \\
-0.966\end{array}$ & $\begin{array}{l}-0.199 \\
-0.845\end{array}$ & $\begin{array}{l}0.816 \\
-0.83\end{array}$ & $\begin{array}{l}0.719 \\
-2.428\end{array}$ & -0.975 & -0.883 \\
\hline-0.209 & $\begin{array}{l}-0.811 \\
-1.392\end{array}$ & $\begin{array}{l}-0.512 \\
-0.586\end{array}$ & $\begin{array}{l}-2.246 \\
-1.209\end{array}$ & $\begin{array}{l}-2.543 \\
-1.232\end{array}$ & $\begin{array}{l}-0.692 \\
-0.36\end{array}$ & $\begin{array}{l}-0.177 \\
-0.412\end{array}$ & $\begin{array}{l}-0.083 \\
-0.979\end{array}$ & $\begin{array}{l}-0.625 \\
-1.876\end{array}$ & $\begin{array}{l}0.627 \\
-0.244\end{array}$ & $\begin{array}{l}1.194 \\
-0.514\end{array}$ & $\begin{array}{l}0.879 \\
-4.058\end{array}$ & $\begin{array}{l}-0.434 \\
-3.561\end{array}$ & -0.964 \\
\hline-0.653 & $\begin{array}{l}-0.392 \\
-0.69\end{array}$ & $\begin{array}{l}-0.373 \\
-1.37\end{array}$ & $\begin{array}{l}-1.954 \\
-0.718\end{array}$ & $\begin{array}{l}-1.927 \\
-0.735\end{array}$ & $\begin{array}{l}-1.618 \\
-0.058\end{array}$ & $\begin{array}{l}-0.259 \\
-1.128\end{array}$ & $\begin{array}{l}-0.368 \\
-0.685\end{array}$ & $\begin{array}{l}-0.099 \\
-0.217\end{array}$ & $\begin{array}{l}1.079 \\
-0.619\end{array}$ & $\begin{array}{l}0.209 \\
-1.335\end{array}$ & $\begin{array}{l}0.618 \\
-2.626\end{array}$ & $\begin{array}{l}-0.789 \\
-5.04\end{array}$ & $\begin{array}{l}-0.876 \\
-2.438\end{array}$ \\
\hline-0.691 & $\begin{array}{l}-0.217 \\
-0.583 \\
-1.042\end{array}$ & $\begin{array}{l}-0.264 \\
-1.438\end{array}$ & $\begin{array}{l}-1.122 \\
-1.169\end{array}$ & $\begin{array}{l}-0.704 \\
-1.025\end{array}$ & $\begin{array}{l}-1.806 \\
-1.179\end{array}$ & $\begin{array}{l}-0.363 \\
-1.155\end{array}$ & $\begin{array}{l}-0.385 \\
-0.691\end{array}$ & $\begin{array}{l}0.018 \\
-0.583\end{array}$ & $\begin{array}{l}-0.608 \\
-1.697\end{array}$ & $\begin{array}{l}-0.72 \\
-0.916\end{array}$ & $\begin{array}{l}-1.094 \\
-2.186\end{array}$ & $\begin{array}{l}-1 . \\
-3 .\end{array}$ & $\begin{array}{l}-0.812 \\
-4.142\end{array}$ \\
\hline-0.319 & $\begin{array}{l}-0.567 \\
-1.378 \\
-1.493\end{array}$ & $\begin{array}{l}-0.474 \\
-1.054 \\
-2.379\end{array}$ & $\begin{array}{l}-0.712 \\
-0.825\end{array}$ & $\begin{array}{l}-0.658 \\
-0.98\end{array}$ & $\begin{array}{l}-0.627 \\
-1.915\end{array}$ & $\begin{array}{l}-0.408 \\
-2.162\end{array}$ & $\begin{array}{l}-0.438 \\
-1.67\end{array}$ & $\begin{array}{l}-0.379 \\
-1.146\end{array}$ & $\begin{array}{l}-1.73 \\
-0.358\end{array}$ & $\begin{array}{l}-1.15 \\
-1.778\end{array}$ & $\begin{array}{l}-1.287 \\
-6.914\end{array}$ & $\begin{array}{l}-0.648 \\
-0.849\end{array}$ & $\begin{array}{l}-1.246 \\
-0.943\end{array}$ \\
\hline-0.504 & $\begin{array}{l}-0.307 \\
-0.864 \\
-0.009\end{array}$ & $\begin{array}{l}-0.36 \\
-0.702 \\
-2.463\end{array}$ & $\begin{array}{l}-0.504 \\
-1.114 \\
-1.633\end{array}$ & $\begin{array}{l}-0.314 \\
-1.127\end{array}$ & $\begin{array}{l}-0.83 \\
-1.849\end{array}$ & $\begin{array}{l}-0.452 \\
-0.916\end{array}$ & $\begin{array}{l}-0.294 \\
-0.975\end{array}$ & $\begin{array}{l}-0.359 \\
-1.47\end{array}$ & $\begin{array}{l}-1.396 \\
-1.627\end{array}$ & $\begin{array}{l}-1.289 \\
-0.165\end{array}$ & $\begin{array}{l}-0.284 \\
-5.859\end{array}$ & $\begin{array}{l}-1.439 \\
-0.89\end{array}$ & $\begin{array}{l}-0.917 \\
-0.432\end{array}$ \\
\hline-0.20 & $\begin{array}{l}0 \\
-1.53 \\
-1.004\end{array}$ & $\begin{array}{l}-0.156 \\
-1.198 \\
-1.422\end{array}$ & $\begin{array}{l}-0.048 \\
-0.969 \\
-0.718\end{array}$ & $\begin{array}{l}-0.058 \\
-1.11 \\
-1.678\end{array}$ & $\begin{array}{l}-0.12 \\
-0.414\end{array}$ & $\begin{array}{l}-0.478 \\
-1.162\end{array}$ & $\begin{array}{l}-0.601 \\
-0.85\end{array}$ & $\begin{array}{l}-0.609 \\
-2.591\end{array}$ & $\begin{array}{l}-0.104 \\
-0.352\end{array}$ & $\begin{array}{l}0.321 \\
-2.748\end{array}$ & $\begin{array}{l}0.31 \\
-1.208\end{array}$ & $\begin{array}{l}95 \\
16\end{array}$ & $\begin{array}{l}-1.232 \\
-1.695\end{array}$ \\
\hline 0.046 & $\begin{array}{l}-0.119 \\
-0.833 \\
-1.614\end{array}$ & $\begin{array}{l}-0.12 \\
-0.825 \\
-0.863\end{array}$ & $\begin{array}{l}-0.106 \\
-1.221 \\
-1.245\end{array}$ & $\begin{array}{l}-0.115 \\
-1.164 \\
-2.81\end{array}$ & $\begin{array}{l}0.02 \\
-1.012 \\
-1.487\end{array}$ & $\begin{array}{l}-0.552 \\
-0.576\end{array}$ & $\begin{array}{l}-0.433 \\
-0.769\end{array}$ & $\begin{array}{l}-0.542 \\
-2.206\end{array}$ & $\begin{array}{l}0.512 \\
-2.971\end{array}$ & $\begin{array}{l}0.057 \\
-0.697\end{array}$ & $\begin{array}{l}0.032 \\
-1.203\end{array}$ & $\begin{array}{l}61 \\
36\end{array}$ & $\begin{array}{l}-1.046 \\
-1.022\end{array}$ \\
\hline 0.373 & $\begin{array}{l}0.289 \\
-0.994 \\
-1.223\end{array}$ & $\begin{array}{l}0.364 \\
-0.669 \\
-1.465\end{array}$ & $\begin{array}{l}0.248 \\
-0.667 \\
-1.411\end{array}$ & $\begin{array}{l}0.175 \\
-0.768 \\
-3.435\end{array}$ & $\begin{array}{l}0.159 \\
-0.947 \\
-3.993\end{array}$ & $\begin{array}{l}-0.432 \\
-0.71 \\
-1.101\end{array}$ & $\begin{array}{l}-0.365 \\
-0.745\end{array}$ & $\begin{array}{l}-0.307 \\
-1.502\end{array}$ & $\begin{array}{l}-0.518 \\
-1.911\end{array}$ & $\begin{array}{l}-0.492 \\
-1.564\end{array}$ & $\begin{array}{l}-0.404 \\
-1.74\end{array}$ & $\begin{array}{l}-1.314 \\
-0.962\end{array}$ & $\begin{array}{l}-1.1 \\
-1.046\end{array}$ \\
\hline-0.446 & $\begin{array}{l}-0.331 \\
-0.609 \\
-1.23\end{array}$ & $\begin{array}{l}-0.445 \\
-0.77 \\
-1.006\end{array}$ & $\begin{array}{l}-0.263 \\
-0.642 \\
0.574\end{array}$ & $\begin{array}{l}-0.144 \\
-0.698 \\
-2.705\end{array}$ & $\begin{array}{l}-0.432 \\
-1.332 \\
-2.445\end{array}$ & $\begin{array}{l}-0.367 \\
-0.973 \\
-1.839\end{array}$ & $\begin{array}{l}-0.353 \\
-0.779 \\
-0.942\end{array}$ & $\begin{array}{l}-0.239 \\
-1.028\end{array}$ & $\begin{array}{l}-1.79 \\
-1.257\end{array}$ & $\begin{array}{l}-1.147 \\
-1.402\end{array}$ & $\begin{array}{l}-1.162 \\
-2.48\end{array}$ & $\begin{array}{l}-0.645 \\
-1.459\end{array}$ & $\begin{array}{l}-0.644 \\
-1.347\end{array}$ \\
\hline-0.158 & $\begin{array}{l}-0.417 \\
-0.941 \\
-1.409\end{array}$ & $\begin{array}{l}-0.24 \\
-0.528 \\
-0.997\end{array}$ & $\begin{array}{l}-0.906 \\
-0.936 \\
-1.259\end{array}$ & $\begin{array}{l}-0.968 \\
-0.949 \\
-1.701\end{array}$ & $\begin{array}{l}-0.28 \\
-0.537 \\
-2.376\end{array}$ & $\begin{array}{l}-0.209 \\
-0.589 \\
-2.244\end{array}$ & $\begin{array}{l}-0.166 \\
-0.69 \\
-2.134\end{array}$ & $\begin{array}{l}-0.419 \\
-2.171 \\
-1.07\end{array}$ & $\begin{array}{l}-0.325 \\
-1.423\end{array}$ & $\begin{array}{l}-0.467 \\
-0.295\end{array}$ & $\begin{array}{l}-0.418 \\
-1.67\end{array}$ & $\begin{array}{l}-0.675 \\
-2.263\end{array}$ & $\begin{array}{l}-0.751 \\
-1.095\end{array}$ \\
\hline-0.455 & $\begin{array}{l}-0.184 \\
-0.638 \\
-0.789\end{array}$ & $\begin{array}{l}-0.181 \\
-1.081 \\
-1.43\end{array}$ & $\begin{array}{l}-0.593 \\
-0.664 \\
-0.553\end{array}$ & $\begin{array}{l}-0.455 \\
-0.649 \\
-2.143\end{array}$ & $\begin{array}{l}-0.852 \\
-0.548 \\
-1.719\end{array}$ & $\begin{array}{l}-0.289 \\
-0.416 \\
-2.17\end{array}$ & $\begin{array}{l}-0.365 \\
-0.408 \\
-2.223\end{array}$ & $\begin{array}{l}-0.04 \\
-0.614 \\
-1.46\end{array}$ & $\begin{array}{l}-0.497 \\
-1.121 \\
-0.845\end{array}$ & $\begin{array}{l}-0.327 \\
-1.944\end{array}$ & $\begin{array}{l}-0.249 \\
-1.495\end{array}$ & $\begin{array}{l}-0.778 \\
-1.162\end{array}$ & $\begin{array}{l}-0.768 \\
-1.292\end{array}$ \\
\hline-0.415 & $\begin{array}{l}-1.615 \\
-1.662 \\
-1.049\end{array}$ & $\begin{array}{l}-1.187 \\
-0.748 \\
-2.361\end{array}$ & $\begin{array}{l}-1.074 \\
-1.081 \\
-0.591\end{array}$ & $\begin{array}{l}-1.263 \\
-1.157 \\
-1.354\end{array}$ & $\begin{array}{l}-0.372 \\
-0.885 \\
-0.914\end{array}$ & $\begin{array}{l}-0.581 \\
-1.035 \\
-0.814\end{array}$ & $\begin{array}{l}-0.532 \\
-0.88 \\
-1.34\end{array}$ & $\begin{array}{l}-1.218 \\
-2.396 \\
-1.083\end{array}$ & $\begin{array}{l}-0.8 \\
-0.097 \\
-0.92\end{array}$ & $\begin{array}{l}-1.12 \\
-0.659 \\
-0.469\end{array}$ & $\begin{array}{l}-1.368 \\
-2.128\end{array}$ & $\begin{array}{l}-0.44 \\
-1.593\end{array}$ & $\begin{array}{l}-1.228 \\
-1.466\end{array}$ \\
\hline-1.475 & $\begin{array}{l}-0.688 \\
-0.752 \\
-0.402\end{array}$ & $\begin{array}{l}-0.931 \\
-1.027 \\
-0.377\end{array}$ & $\begin{array}{l}-0.96 \\
-1.077 \\
-0.115\end{array}$ & $\begin{array}{l}-0.782 \\
-0.984 \\
-0.51\end{array}$ & $\begin{array}{l}-1.41 \\
-0.265 \\
-1.096\end{array}$ & $\begin{array}{l}-0.826 \\
-0.331 \\
-0.878\end{array}$ & $\begin{array}{l}-0.754 \\
-0.354 \\
-0.525\end{array}$ & $\begin{array}{l}-0.521 \\
-0.479 \\
-1.002\end{array}$ & $\begin{array}{l}-0.239 \\
-1.781 \\
-0.321\end{array}$ & $\begin{array}{l}-0.744 \\
-0.471 \\
0.348\end{array}$ & $\begin{array}{l}-0.314 \\
-1.088 \\
0.099\end{array}$ & $\begin{array}{l}-1.952 \\
-1.424\end{array}$ & $\begin{array}{l}-1.134 \\
-0.24\end{array}$ \\
\hline
\end{tabular}




\begin{tabular}{|c|c|c|c|c|c|c|c|c|c|c|c|c|c|}
\hline \multirow[t]{3}{*}{-1.171} & -0.722 & -0.855 & -0.58 & -0.522 & -0.898 & -0.795 & -0.878 & -0.362 & -1.833 & -0.789 & -0.455 & -1.169 & -1.033 \\
\hline & -0.899 & -1.144 & -0.754 & -0.696 & -0.73 & -0.317 & -0.357 & -0.373 & -0.726 & -1.04 & -1.745 & -0.594 & -0.354 \\
\hline & -0.003 & -0.501 & -0.791 & -0.795 & -0.634 & -0.842 & -0.676 & -0.257 & -0.5 & 0.556 & 0.654 & 0.151 & \\
\hline \multirow[t]{3}{*}{-0.652} & -0.57 & -0.595 & -1.257 & -1.207 & -1.057 & -0.495 & -0.525 & -0.359 & -1.275 & -1.099 & -1.355 & -1.421 & -1.32 \\
\hline & -1.467 & -0.965 & -0.589 & -0.527 & -3.099 & -2.592 & -2.818 & -0.218 & -0.318 & -0.288 & 0.183 & -0.126 & 0.123 \\
\hline & 0.175 & -0.689 & 0.025 & -0.196 & -0.227 & -0.071 & 0.914 & -2.16 & -2.54 & 0.079 & 0.55 & 0.783 & 0.706 \\
\hline \multirow[t]{4}{*}{-0.708} & -0.341 & -0.474 & -1.138 & -1.096 & -1.411 & -0.516 & -0.59 & -0.177 & -1.962 & -0.577 & -1.387 & -1.547 & -1.152 \\
\hline & -0.725 & -1.745 & -1.218 & -0.847 & -5.462 & -2.838 & -2.958 & -0.185 & -0.424 & -0.347 & -0.412 & -0.237 & 0.025 \\
\hline & -0.017 & -0.672 & 0.263 & -0.248 & -0.214 & -0.446 & 0.261 & 2.013 & 3.146 & 0.05 & -0.93 & 0.52 & 3.908 \\
\hline & 1.444 & & & & & & & & & & & & \\
\hline \multirow[t]{4}{*}{-0.247} & -0.538 & -0.417 & -1.522 & -1.491 & -0.915 & -0.298 & -0.215 & -0.462 & -0.998 & -1.846 & -0.756 & -0.493 & -1.43 \\
\hline & -2.082 & -0.718 & -1.035 & -1.042 & -2.847 & -3.896 & -3.656 & -0.335 & -0.103 & -0.184 & -0.128 & -0.227 & -0.03 \\
\hline & 0.066 & -0.764 & -0.244 & -0.18 & -0.222 & -0.382 & 0.542 & 1.496 & 1.972 & -2.048 & 0.805 & -0.007 & 3.573 \\
\hline & 1.796 & 1.97 & & & & & & & & & & & \\
\hline \multirow[t]{4}{*}{-0.252} & -0.536 & -0.401 & -0.06 & 0.041 & -0.072 & -0.17 & -0.181 & -0.3 & 0.412 & 0.511 & 0.527 & -0.252 & -0.397 \\
\hline & -0.625 & -0.211 & -0.178 & -0.26 & 0.229 & -0.283 & -0.124 & -0.787 & -0.186 & -0.116 & -3.03 & -2.167 & -0.811 \\
\hline & -0.436 & -1.389 & -2.459 & -0.445 & -0.524 & -0.155 & -2.343 & -1.191 & -0.939 & 0.071 & 0.484 & 0.44 & -1.353 \\
\hline & -1.363 & -0.537 & -1.083 & & & & & & & & & & \\
\hline \multirow[t]{4}{*}{-0.505} & -0.221 & -0.28 & -0.115 & -0.15 & 0.263 & -0.179 & -0.245 & -0.072 & 0.656 & 0.326 & 0.446 & -0.455 & -0.42 \\
\hline & -0.27 & -0.208 & -0.206 & -0.18 & 0.027 & 0.213 & 0.127 & -0.181 & -0.322 & -0.227 & -3.527 & -0.848 & -1.243 \\
\hline & -0.971 & -3.257 & -0.775 & -0.372 & -0.262 & -0.139 & -2.527 & -0.717 & -0.787 & 0.257 & -0.024 & 1.648 & -1.109 \\
\hline & -0.543 & -1.019 & -0.782 & -0.617 & & & & & & & & & \\
\hline \multirow[t]{4}{*}{-0.255} & -0.234 & -0.193 & -0.15 & -0.13 & -0.227 & -0.158 & -0.175 & -0.14 & -0.318 & -0.084 & -0.012 & -0.503 & -0.502 \\
\hline & -0.497 & -0.284 & -0.339 & -0.251 & -0.423 & -0.351 & -0.241 & -0.554 & -0.186 & -0.091 & 0.466 & -0.084 & 0.126 \\
\hline & -0.344 & -3.117 & -2.265 & -0.162 & -0.224 & -0.331 & -0.455 & -0.107 & -0.034 & 0.202 & 0.252 & 0.209 & -0.714 \\
\hline & -0.851 & -0.939 & -1.396 & -0.596 & -1.055 & & & & & & & & \\
\hline \multirow[t]{4}{*}{-0.187} & -0.092 & -0.125 & 0.133 & 0.206 & 0.042 & -0.075 & -0.082 & -0.058 & -0.14 & 0.312 & 0.304 & 0.173 & -0.073 \\
\hline & -0.136 & 0.109 & 0.076 & 0.003 & -0.139 & 0.099 & -0.101 & -0.56 & -0.181 & -0.022 & -0.849 & -0.526 & -0.256 \\
\hline & -0.354 & -0.761 & -0.328 & -0.355 & -0.768 & -0.592 & -1.837 & -0.415 & -0.548 & -0.468 & 0.176 & 0.145 & -0.331 \\
\hline & -0.65 & -0.073 & 0.145 & -0.778 & -0.948 & -0.043 & & & & & & & \\
\hline \multirow[t]{4}{*}{-0.109} & -0.21 & -0.167 & 0.024 & 0.133 & 0.135 & -0.055 & -0.069 & -0.081 & 0.265 & 0.444 & 0.34 & -0.026 & 0.072 \\
\hline & 0.051 & 0.048 & 0.116 & 0.079 & 0.316 & 0.239 & 0.06 & -0.474 & -0.117 & -0.078 & -0.386 & -0.443 & -0.191 \\
\hline & 0.164 & -0.376 & -0.006 & -0.744 & -0.41 & -0.426 & -2.541 & -0.226 & -0.05 & 0.027 & 0.287 & 0.258 & -0.039 \\
\hline & -0.027 & 0.277 & -0.554 & 0.801 & -0.796 & -0.032 & 0.041 & & & & & & \\
\hline \multirow[t]{4}{*}{0.118} & 0.089 & 0.125 & 0.184 & 0.249 & -0.277 & 0.06 & 0.037 & 0.115 & -1.644 & 0.167 & 0.236 & -0.176 & -0.182 \\
\hline & -0.188 & -0.112 & -0.094 & -0.052 & -1.017 & 0.123 & -0.22 & -0.137 & 0.12 & 0.064 & -1.388 & -0.125 & -0.079 \\
\hline & -0.03 & -0.905 & -0.299 & -0.35 & -0.318 & -0.568 & -0.242 & -0.06 & 0.045 & 1.092 & 1.483 & 2.364 & 0.741 \\
\hline & -0.096 & 0.087 & -2.718 & -2.413 & -1.116 & -0.007 & 0.269 & -0.729 & & & & & \\
\hline \multirow[t]{4}{*}{0.663} & 0.183 & 0.372 & 0.155 & -0.009 & -0.036 & 0.133 & 0.108 & 0.137 & -1.412 & -0.169 & -0.067 & -1.317 & -0.497 \\
\hline & -0.141 & -0.439 & -0.359 & -0.231 & -1.791 & -0.301 & -0.687 & -0.227 & -0.875 & -0.114 & -1.823 & -0.242 & 0.324 \\
\hline & -0.836 & -1.088 & 0.044 & -0.343 & -0.667 & -0.917 & -2.296 & 0.158 & 1.797 & -1.659 & -0.737 & -0.242 & 2.208 \\
\hline & -3.664 & -1.321 & -0.307 & 0.026 & -0.76 & -0.89 & 0.385 & -4.936 & -4.563 & & & & \\
\hline \multirow[t]{4}{*}{0.021} & 0.03 & 0.05 & 0.376 & 0.352 & 0.185 & -0.078 & -0.177 & -0.002 & -0.048 & 0.174 & 0.478 & 0.055 & -0.404 \\
\hline & -0.406 & -0.711 & -0.015 & -0.057 & 0.221 & -0.332 & -0.1 & -0.441 & 0.238 & 0.903 & -0.543 & -0.659 & -0.518 \\
\hline & -0.547 & 0.431 & 0.133 & -0.486 & -0.105 & -0.317 & -0.277 & 0.196 & -0.34 & 0.193 & 0.024 & -0.132 & 4.17 \\
\hline & 0.023 & 0.351 & -0.022 & -0.055 & 0.094 & 0.354 & 0.45 & -2.234 & -1.111 & -3.475 & & & \\
\hline
\end{tabular}




\begin{tabular}{|c|c|c|c|c|c|c|c|c|c|c|c|c|c|}
\hline \multirow[t]{4}{*}{0.037} & 0.363 & 0.339 & 0.605 & 0.706 & 0.741 & 0.21 & 0.121 & 0.486 & 0.472 & 0.557 & 0.433 & -0.213 & -0.04 \\
\hline & 0.099 & -0.098 & 1.083 & 1.11 & 0.415 & -0.289 & -0.281 & -0.48 & -0.15 & 0.371 & -0.187 & -0.178 & 1.107 \\
\hline & 0.673 & -0.52 & 0.048 & -0.35 & -0.249 & -0.189 & 0.927 & -0.107 & 0.765 & -1.213 & 0.757 & 0.037 & 0.366 \\
\hline & 0.225 & 1.303 & -0.421 & -0.47 & -0.028 & 0.562 & 0.821 & -0.688 & 0.211 & -1.793 & 0.862 & & \\
\hline \multirow[t]{4}{*}{0.127} & -0.076 & 0.026 & -0.099 & 0.009 & -0.403 & -0.209 & -0.272 & -0.073 & -1.746 & 0.181 & 0.264 & -0.406 & -0.445 \\
\hline & -0.318 & -0.352 & 0.261 & 0.378 & -1.058 & 0.735 & 0.343 & -0.503 & -0.184 & -0.046 & -0.32 & -1.284 & -0.592 \\
\hline & -0.458 & -1.189 & -0.096 & -0.46 & -0.302 & -0.373 & -0.913 & 0.042 & 0.371 & -0.591 & 0.604 & 0.364 & 0.044 \\
\hline & -0.655 & -0.031 & -0.292 & 0.022 & -0.629 & -0.296 & 0.349 & -3.058 & -5.839 & -2.683 & 0.667 & -0.767 & \\
\hline \multirow[t]{4}{*}{0.484} & 0.183 & 0.356 & 0.092 & 0.154 & -0.339 & 0.303 & 0.266 & 0.231 & -1.918 & 0.182 & 0.196 & -0.734 & -0.487 \\
\hline & -0.276 & -0.177 & 0.267 & 0.157 & -1.459 & -0.061 & -0.717 & -0.073 & -0.293 & -0.122 & -1.741 & 0.129 & 1.383 \\
\hline & 0.554 & -1.44 & 0.108 & -0.44 & -0.249 & -0.504 & -0.678 & -0.075 & 0.273 & -0.529 & 0.69 & 0.605 & -0.673 \\
\hline & -1.331 & -1.082 & -0.591 & -0.442 & -0.747 & -0.498 & 0.138 & -2.513 & -3.882 & -1.103 & 1.752 & -2.766 & -0.602 \\
\hline \multirow[t]{5}{*}{0.028} & 0.439 & 0.43 & 0.443 & 0.271 & 0.926 & 0.186 & 0.13 & 0.603 & 0.404 & 0.055 & -0.11 & -0.025 & 0.01 \\
\hline & 0.208 & -0.116 & 0.633 & 0.593 & 0.865 & -0.454 & -0.197 & -1.093 & 0.064 & 0.5 & -0.457 & 0.37 & 1.354 \\
\hline & 1.404 & -0.408 & -0.18 & -0.189 & 0.074 & -0.267 & 0.083 & 0.004 & 1.028 & 0.347 & 1.43 & 0.462 & 0.482 \\
\hline & -0.016 & 0.476 & -0.624 & -0.294 & -0.359 & 0.246 & 0.398 & -0.151 & 0.432 & -1.635 & 5.23 & 0.909 & 1.578 \\
\hline & 0.656 & & & & & & & & & & & & \\
\hline \multirow[t]{5}{*}{0.402} & 0.296 & 0.478 & -0.008 & 0.129 & 0.514 & 0.878 & 1.272 & 0.444 & 0.092 & 0.267 & -0.03 & -0.136 & -0.973 \\
\hline & -1.065 & -0.601 & -0.174 & -0.53 & -0.265 & -0.815 & -1.058 & -1.065 & 0.871 & 0.176 & -0.26 & 0.855 & 1.711 \\
\hline & 1.629 & -1.199 & 1.302 & -0.83 & -0.109 & 0.264 & -1.1 & 0.189 & 0.708 & -0.574 & 1.126 & 1.385 & -1.047 \\
\hline & -0.817 & 0.185 & -1.385 & -0.676 & -0.068 & -0.826 & -0.614 & -1.009 & 2.109 & -2.088 & 1.593 & -0.173 & 5.118 \\
\hline & 2.084 & 0.192 & & & & & & & & & & & \\
\hline \multirow[t]{5}{*}{0.22} & 1.106 & 0.99 & 0.532 & 0.432 & 0.923 & 0.658 & 0.674 & 1.581 & 0.289 & 0.317 & 0.366 & 0.138 & -0.78 \\
\hline & -1.344 & -0.178 & -0.647 & -0.848 & 0.699 & -1.1 & -0.911 & 1.295 & -0.076 & -0.01 & -0.2 & -0.51 & 1.925 \\
\hline & 3.158 & -0.229 & 0.173 & -0.062 & -0.042 & -0.082 & 0.004 & -0.102 & 1.464 & -0.088 & 2.386 & 0.728 & 0.147 \\
\hline & 0.376 & -0.676 & -0.154 & -0.083 & -0.173 & -0.015 & 0.078 & -0.535 & -0.159 & -1.018 & 2.876 & 1.317 & 2.082 \\
\hline & 4.261 & 2.533 & 1.978 & & & & & & & & & & \\
\hline \multirow[t]{5}{*}{0.393} & 0.649 & 1.093 & 0.561 & 0.778 & 0.139 & -0.502 & -0.072 & 0.098 & -0.946 & 0.303 & 0.725 & 0.379 & -0.473 \\
\hline & -0.441 & -0.203 & 0.328 & 0.377 & 0.596 & -0.873 & 0.049 & -0.834 & 1.576 & -1.361 & -0.473 & 0.201 & 1.008 \\
\hline & 2.922 & -1.142 & 1.738 & 0.053 & 0.389 & -0.249 & 0.468 & 0.05 & 0.963 & -0.76 & 1.894 & 1.836 & 1.884 \\
\hline & 0.612 & -0.093 & 0.794 & 0.333 & 0.097 & -0.147 & 1.411 & 0.069 & -0.209 & -0.906 & 4.229 & 2.355 & 1.993 \\
\hline & 2.485 & 2.478 & 0.554 & 1.485 & & & & & & & & & \\
\hline
\end{tabular}

\section{3 'GHZ'}

58 vib $64.4227-18.6381$

59 vib $79.602-15.1081$

60 vib $81.0111-12.5151$ 